\title{
HOBBY-EBERLY TELESCOPE OBSERVATIONS OF THE DARK HALO IN NGC 821*
}

\author{
Amy D. Forestell and Karl Gebhardt \\ Department of Astronomy, University of Texas at Austin, C1400, 1 University Station, Austin, TX 78712, USA; \\ amydove@astro.as.utexas.edu, gebhardt@astro.as.utexas.edu \\ Received 2007 May 2; accepted 2010 April 19; published 2010 May 18
}

\begin{abstract}
We present stellar line-of-sight velocity distributions (LOSVDs) of elliptical galaxy NGC 821 obtained to approximately $100^{\prime \prime}$ (over two effective radii) with long-slit spectroscopy from the Hobby-Eberly Telescope. Our measured stellar LOSVDs are larger than the planetary nebulae measurements at similar radii. We fit axisymmetric orbit-superposition models with a range of dark halo density profiles, including two-dimensional kinematics at smaller radii from SAURON data. Within our assumptions, the best-fitted model gives a total enclosed mass of $2.0 \times 10^{11} M_{\odot}$ within $100^{\prime \prime}$, with an accuracy of $2 \%$; this mass is equally divided between halo and stars. At $1 R_{e}$, the best-fitted dark matter halo accounts for $13 \%$ of the total mass in the galaxy. This dark halo is inconsistent with previous claims of little to no dark matter halo in this galaxy from planetary nebula measurements. We find that a power-law dark halo with a slope 0.1 is the best-fitted model; both the no dark halo and Navarro-Frenk-White models are worse fits at a greater than $99 \%$ confidence level. NGC 821 does not appear to have the expected dark halo density profile. The internal moments of the stellar velocity distribution show that the model with no dark halo is radially anisotropic at small radii and tangentially isotropic at large radii, while the best-fitted halo models are slightly radially anisotropic at all radii. We test the potential effects of model smoothing and find that there are no effects on our results within the errors. Finally, we run models using the planetary nebula kinematics and assuming our best-fitted halos and find that the planetary nebulae require radial orbits throughout the galaxy.
\end{abstract}

Key words: dark matter - galaxies: elliptical and lenticular, cD - galaxies: halos - galaxies: individual (NGC 821) - galaxies: kinematics and dynamics

Online-only material: color figures, machine-readable table

\section{INTRODUCTION}

Cold dark matter is now accepted as an integral part of our universe, and recent observations have continued to provide support for its existence (Komatsu et al. 2009). Part of the picture of the universe is that galaxies are surrounded by massive dark matter halos in which they formed (White \& Rees 1978; Blumenthal et al. 1984). Recently, cosmological simulations have become detailed enough to reach the level of individual galaxy formation (Naab et al. 2007; Governato et al. 2007), and comparisons with data can help further constrain cosmological theory (Ostriker \& Steinhardt 2003). Indeed, spiral galaxy rotation curves are one of the strongest pieces of observational evidence for the existence of dark matter (van Albada et al. 1985; Persic et al. 1996; Sofue \& Rubin 2001). It is also important to study the dark halo structure of elliptical galaxies because of their different formation and evolution. However, it is more difficult to measure dark matter in elliptical galaxies because of a lack of tracers at large radii where dark matter is thought to dominate. The best way to measure the underlying gravitational potential is to use kinematics from the stellar population, but this has been limited due to the faintness of stellar light in the outer regions of galaxies (Gerhard et al. 2001). Dark matter in elliptical galaxies has therefore been studied in other ways, such as via X-ray emission (Loewenstein \& White 1999; Mathews \& Brighenti 2003), gravitational lensing (Keeton 2001; Mandelbaum et al. 2009), and using individual stellar velocities as in nearby dwarf spheroidals (Mateo 1998;

\footnotetext{
* Based on observations obtained with the Hobby-Eberly Telescope, which is a joint project of the University of Texas at Austin, the Pennsylvania State University, Stanford University, Ludwig-Maximilians-Universität München, and Georg-August-Universität Göttingen.
}

Kleyna et al. 2002). In order to study a more representative sample of galaxies, tracers such as globular clusters (Zepf et al. 2000; Pierce et al. 2006) and planetary nebulae (Méndez et al. 2001; Romanowsky et al. 2003; Coccato et al. 2009) are used to probe the outer parts of elliptical galaxies, though it is difficult to get a significant sample size. Additional issues arise with these tracers, as discussed below, such as understanding their radial profile. With larger telescopes we are now able to measure stellar kinematics from integrated light to larger radii, thus closing the gap between stars and the large-radii tracers.

Meanwhile, dynamical models of galaxies have also improved. Rather than previous spherical models that use analytic distribution functions (DFs; Gerhard et al. 2001), orbit-based axisymmetric models are now available. These fully general models, based on the technique of Schwarzschild (1979), provide detailed information on the orbital structure of the galaxy, including the DF and its projections, such as velocity anisotropy. Orbit-based models are now frequently applied to galaxies for studies of both dark halos and central black holes (Rix et al. 1997; van der Marel et al. 1998; Cretton et al. 1999; Gebhardt et al. 2000; Cappellari et al. 2002; Verolme et al. 2002; Gebhardt et al. 2003; Thomas et al. 2005; Gebhardt \& Thomas 2009).

The elliptical galaxy NGC 821 is an example in which the use of large-radii tracers has provided an intriguing result. Romanowsky et al. (2003) study the dark halo of NGC 821 using approximately 100 planetary nebula velocities and find small line-of-sight (LOS) velocity dispersions that are consistent with little or no dark halo. Dekel et al. (2005) use disk galaxy merger simulations to show that large anisotropies can be created in the resulting elliptical galaxies and that this anisotropy in combination with the different density profile of a young population could explain how the low dispersions from planetary 
nebulae measurements are also consistent with typical dark matter halos. Our study uses deep long-slit spectroscopy of NGC 821 from the $9.2 \mathrm{~m}$ Hobby-Eberly Telescope (HET) to obtain stellar kinematics to greater than 2 effective radii in hopes of further constraining the dark halo of this galaxy.

Weijmans et al. (2009) model NGC 821 using data from SAURON, both at small radii (which we include in our analysis) and newer data at large radii. We find similar results both for the kinematics and for the dark halo properties. Comparison between the two studies is presented in their paper and in this paper.

NGC 821 is classified as an E6? (de Vaucouleurs et al. 1991). It has disky isophotes (Lauer 1985; Bender et al. 1988) and a power-law central surface brightness profile (Ravindranath et al. 2001). The blue absolute magnitude is -20.27 (Trager et al. 2000). We use a distance of $23.44 \mathrm{Mpc}$ taken from Cappellari et al. (2006), which adjusts the Tonry et al. (2001) values for the new Cepheids zero point of Freedman et al. (2001). NGC 821 is not detected in $\mathrm{H} \alpha$ (Macchetto et al. 1996) or in O III (Sarzi et al. 2006). Point source and diffuse X-ray emission have been detected but there is no evidence for hot gas (Pellegrini et al. 2007a, 2007b). NGC 821 is considered a fast rotator (Cappellari et al. 2007; Emsellem et al. 2007). Proctor et al. (2005) find that NGC 821 has very strong age and metallicity gradients, from $\sim 4$ Gyr and 3 times solar in the center to $\sim 12$ Gyr and less than $\frac{1}{3}$ solar at $1 R_{e}$. They conclude that NGC 821 has experienced a recent ( $\sim 1-4$ Gyr ago) burst of star formation, most likely from in situ gas and perhaps triggered by the accretion of a small satellite galaxy. This may be an indication that there are young planetary nebulae in this galaxy.

Section 2 describes the observations and data reduction; in Section 3, we describe the kinematic extraction; the dynamical models are described in Section 4; we present our results in Section 5 and give conclusions in Section 6.

\section{OBSERVATIONS AND DATA REDUCTION}

Long-slit spectra were taken with the low-resolution spectrograph (Hill et al. 1998) on the HET. We use the g2 grism and 1" by $4^{\prime}$ slit over the wavelength range 4300-7300 A. This setup gives a resolving power of 1300 or an FWHM resolution of about $230 \mathrm{~km} \mathrm{~s}^{-1}$. Measurements of night skyline widths show that we can measure dispersions to about $110 \mathrm{~km} \mathrm{~s}^{-1}$. The CCD frame (binned $2 \times 2$ ) has a plate scale of 0.47 pixel $^{-1}$ spatially and $2 \AA$ pixel $^{-1}$ spectrally. The gain is $1.832 e^{-} \mathrm{ADU}^{-1}$ and readout noise is $5.10 e^{-}$. We use the Schott Glass blocking filter GG385, which has a half-power point of the transmission around $385 \mathrm{~nm}$.

NGC 821 was observed over eight nights in 2003 November for a total exposure time of approximately 5.5 and $2.3 \mathrm{hr}$ on the major and minor axes, respectively. Cadmium and neon calibration lamp exposures and white light illumination flat fields were taken each night.

The data reduction uses standard techniques. First, we overscan correct and trim the images. Then, we apply a flat correction using a normalized flat frame, taken from averaged instrumental flats obtained each night of observations. Next, we rectify the images along the spatial axis using the calibration lamp lines as a reference.

For sky subtraction, we use the region of the slit that is furthest from the galaxy center. Since we only have a $4^{\prime}$ slit, there will be some galaxy light in the region where we select sky. However, the surface brightness profile extends out $350^{\prime \prime}$ so we can accurately calculate the amount of galaxy in our background region. For our last extracted spectrum (at $90^{\prime \prime}$ ), the amount of galaxy light that we are including as background light is about $15 \%$ of the galaxy light for that last extraction. We have run simulations in order to determine whether this amount of contamination has an effect on the extracted kinematics. We take a high signal-to-noise galaxy spectrum and subtract off $15 \%$ of itself and then extract the kinematics. Only for very high signalto-noise ratio $(\mathrm{S} / \mathrm{N})$ does this amount have an effect, and for the $\mathrm{S} / \mathrm{N}$ for this data set (as described below) we find no significant effect.

\section{KINEMATICS}

We extract the spectra in radial bins along the major and minor axes. Because the seeing is approximately $2^{\prime \prime}$, we set the central bins to 5 pixels (2".35). The outer bins are sized to obtain sufficient signal for kinematic analysis. Along the minor axis, the spectra from either side of the galaxy were averaged at each radius before kinematic analysis. Along the major axis, the center of the galaxy was near the edge of the chip so only one side was extracted. Our farthest radial bin extends to $99^{\prime \prime}$ on the major axis and $45^{\prime \prime}$ on the minor axis, corresponding to a $V$-band surface brightness of 21.8 and 23.5 , respectively. There is a faint object centered at $115^{\prime \prime}$ along the major axis that prevents a further radial bin from being used. The radial extent in effective radii depends on the value of $R_{e}$ used. The measured $R_{e}$ of NGC 821 varies throughout the literature: 50" (RC3), 45" (Faber et al. 1989), 39" (Cappellari et al. 2006), 36" (Trager et al. 2000), and 16".7-18.'3 (Bender et al. 1988). For the purpose of this discussion, we adopt an $R_{e}$ of $45^{\prime \prime}$. Thus, our data extend to approximately $1 R_{e}$ along the minor axis and $2 R_{e}$ along the major axis.

We do not flux calibrate the spectra, and thus we remove the continuum in each spectrum. We fit the local continuum by finding the biweight (Beers et al. 1990) in windows as described in Pinkney et al. (2003). The wavelength solution comes from the $\mathrm{Cd}$ and $\mathrm{Ne}$ calibration lamps.

We obtain a nonparametric line-of-sight velocity distribution (LOSVD) by deconvolving the galaxy spectrum with a set of stellar template spectra using the maximum penalized likelihood technique of Gebhardt et al. (2000). Tests of this technique are given in Pinkney et al. (2003). There are 30 evenly spaced velocity bins of $54 \mathrm{~km} \mathrm{~s}^{-1}$ that represent the LOSVD. We vary the height in each bin and the weights of each template star to find the best match to the galaxy spectrum from each radial range. We use nine stellar templates with types ranging from $\mathrm{G}$ dwarf to M giant from Leitherer et al. (1996), convolved to our spectral resolution.

For our kinematic analysis, we use the spectral range 4800-5450 A which matches the wavelength range of our template stars. This region includes the $\mathrm{H} \beta$ and $\mathrm{Mg} b$ lines; however, we exclude the $\mathrm{Mg} b$ region because it is enhanced (Proctor et al. 2005) and our template stars do not provide a proper fit. Barth et al. (2002) show that in pixel-space fitting routines the $\mathrm{Mg} b$ line is sensitive to template mismatch and the details of the fitting procedure. If the $\mathrm{Mg} b$ line is included in the fit, the measured dispersions are falsely high by as much as $20 \%$ to account for the abundance discrepancy. An example fit is shown in Figure 1.

The uncertainty of each velocity bin is obtained from Monte Carlo simulations. We convolve the best-fitted LOSVD and weighted stellar templates to obtain an initial galaxy spectrum. We then generate 100 realizations of the galaxy spectrum by adding Gaussian noise using an estimate of the initial rms. The LOSVD is determined for each realization as described above. 


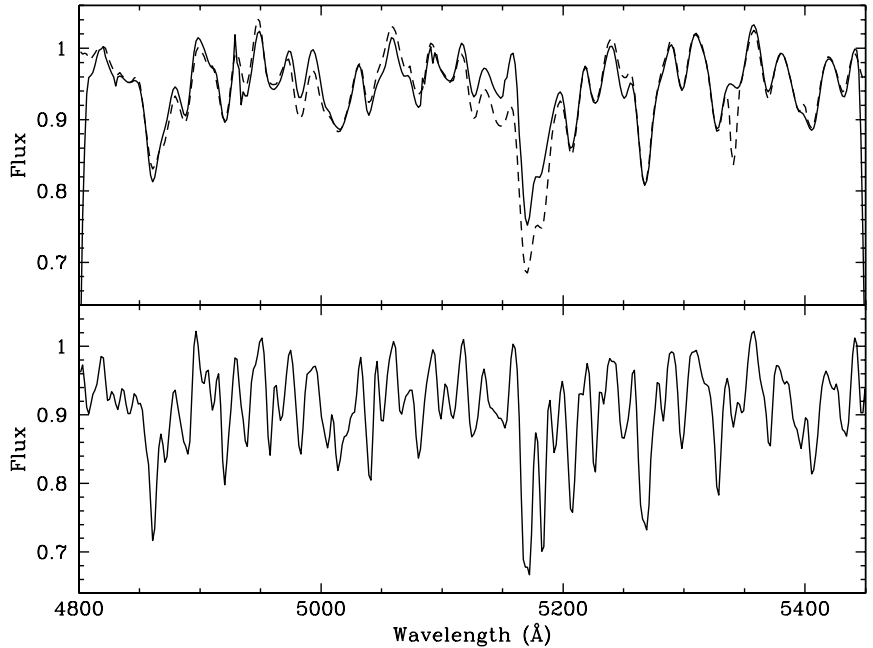

Figure 1. Spectrum of the combined, weighted template stars (lower panel), data from the central bin along the minor axis (dashed line, upper panel), and the template spectrum convolved with the best-fitted LOSVD (solid line, upper panel). The region from $5163 \AA$ to $5228 \AA$ is excluded from the fit.

The distribution of values in each velocity bin of the LOSVD provides an estimate of the $68 \%$ confidence bands. The median value of the dispersion from the 100 realizations compared to the initial dispersion reveals any possible bias in the dispersion measurement.

Although we use the full nonparametric velocity profile in the dynamic modeling, it is useful to compare moments of the distribution. In Figure 2 we plot, from top to bottom, the second moment as measured by $\left.\sqrt{(} V^{2}+\sigma^{2}\right)$, the first four Gauss-Hermite moments (mean velocity $V$, velocity dispersion $\sigma$, asymmetric deviations from Gaussian (similar to skewness) $h_{3}$, and symmetric deviations from Gaussian (similar to kurtosis) $h_{4}$ ). The kinematic data are given in Tables 1 and 2. For comparison, in Figure 2 we also plot data from Pinkney et al. (2003) and Emsellem et al. (2004) extracted in a $1^{\prime \prime}$ slit along the major and minor axes. The second moment of the LOS velocity, $\left(V^{2}+\sigma^{2}\right)^{1 / 2}$, is slightly smaller than the other samples throughout the overlapping region. This may be caused by a slit misalignment (since $V$ will be higher on the major axis) or template fitting difference. Since, however, our models are dominated by SAURON data in the center, this difference is not a major issue. We further run the dynamical models using SAURON data alone and HET data alone and find unbiased results from when using the combined data set.

Our $h_{4}$ values are more negative than the data from the literature. This discrepancy could be attributed to template mismatch (either in the published analysis or in ours); however, we use a wide range of template stars and do not get a different result when more template stars are made available for the fit. It could also be that relying on Gauss-Hermite parameterization causes some differences since there are known correlations, especially with higher-order moments (see Magorrian 2006; Houghton et al. 2006). Since we fit the LOSVD directly in the dynamical models, a better comparison would be with those profiles, as opposed to their moments. The dark halo mass, however, is determined mainly by the radial profile of the second moment, and $h_{4}$ determines mainly the anisotropies. There is certainly some degeneracy between the two parameters, but we find no reason to believe that our $h_{4}$ values are incorrect. There are also kinematic points that have differences which are inconsistent with their reported uncertainties (for example,

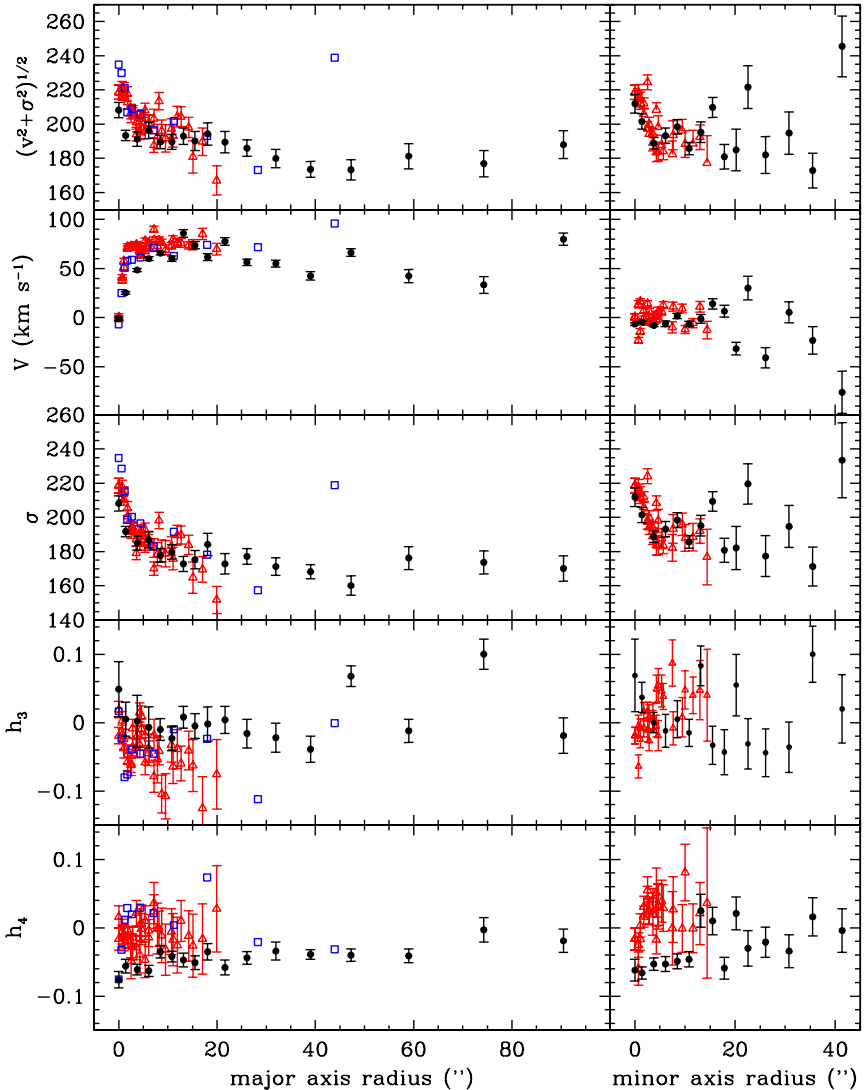

Figure 2. Gauss-Hermite moments of the LOSVDs and the rms LOS velocity: mean velocity $V$, velocity dispersion $\sigma$, asymmetric deviations from Gaussian (skewness) $h_{3}$, and symmetric deviations from Gaussian (kurtosis) $h_{4}$ along the major axis (left panel) and minor axis (right panel) for our data (black filled circles), SAURON (Emsellem et al. 2004, blue open triangles), and Pinkney et al. (2003, red open squares).

(A color version of this figure is available in the online journal.)

Table 1

Major Axis Kinematics

\begin{tabular}{lccccrcrc}
\hline \hline $\begin{array}{c}r \\
(\operatorname{arcsec})\end{array}$ & $\begin{array}{c}v \\
\left(\mathrm{~km} \mathrm{~s}^{-1}\right)\end{array}$ & $\epsilon_{v}$ & $\begin{array}{c}\sigma \\
\left(\mathrm{km} \mathrm{s}^{-1}\right)\end{array}$ & $\epsilon_{\sigma}$ & \multicolumn{1}{c}{$h_{3}$} & $\epsilon_{h_{3}}$ & $h_{4}$ & $\epsilon_{h_{4}}$ \\
\hline 0.00 & -1.05 & 1.65 & 208.24 & 4.43 & 0.049 & 0.040 & -0.076 & 0.012 \\
1.41 & 25.35 & 1.40 & 191.71 & 2.96 & 0.005 & 0.025 & -0.056 & 0.010 \\
3.76 & 48.53 & 1.55 & 185.06 & 4.16 & 0.002 & 0.038 & -0.061 & 0.008 \\
6.11 & 60.13 & 1.89 & 186.95 & 4.73 & -0.007 & 0.030 & -0.063 & 0.008 \\
8.46 & 65.68 & 1.72 & 177.81 & 3.93 & -0.010 & 0.016 & -0.035 & 0.009 \\
10.81 & 60.16 & 2.79 & 179.86 & 4.20 & -0.023 & 0.018 & -0.042 & 0.008 \\
13.16 & 85.90 & 3.80 & 172.81 & 4.40 & 0.008 & 0.016 & -0.047 & 0.010 \\
15.51 & 73.79 & 3.76 & 175.29 & 5.35 & -0.005 & 0.018 & -0.051 & 0.010 \\
18.09 & 61.67 & 3.37 & 184.25 & 6.40 & -0.002 & 0.025 & -0.035 & 0.012 \\
21.62 & 77.70 & 3.91 & 172.87 & 5.93 & 0.004 & 0.020 & -0.058 & 0.011 \\
26.08 & 56.48 & 3.27 & 177.18 & 4.56 & -0.016 & 0.021 & -0.044 & 0.009 \\
31.96 & 55.20 & 3.46 & 171.32 & 5.12 & -0.022 & 0.021 & -0.034 & 0.013 \\
39.01 & 42.58 & 4.29 & 168.27 & 4.21 & -0.039 & 0.019 & -0.039 & 0.007 \\
47.24 & 66.29 & 3.89 & 160.18 & 5.64 & 0.068 & 0.015 & -0.040 & 0.009 \\
58.99 & 42.41 & 6.68 & 176.27 & 6.69 & -0.012 & 0.017 & -0.041 & 0.010 \\
74.26 & 33.40 & 8.50 & 173.74 & 6.79 & 0.100 & 0.022 & -0.003 & 0.018 \\
90.47 & 79.97 & 6.16 & 170.17 & 7.48 & -0.019 & 0.026 & -0.019 & 0.017 \\
& & & & & & & &
\end{tabular}

some of the minor axis points), and the uncertainties may be underestimated for those points. We run halo models without the most discrepant points and still find the same halo results as when they are included.

Proctor et al. (2009) measure the kinematics of NGC 821 using the background galaxy light from Keck DEIMOS 
Table 2

Minor Axis Kinematics

\begin{tabular}{|c|c|c|c|c|c|c|c|c|}
\hline $\begin{array}{c}r \\
(\operatorname{arcsec})\end{array}$ & $\begin{array}{c}v \\
\left(\mathrm{~km} \mathrm{~s}^{-1}\right)\end{array}$ & $\epsilon_{v}$ & $\begin{array}{c}\sigma \\
\left(\mathrm{km} \mathrm{s}^{-1}\right)\end{array}$ & $\epsilon_{\sigma}$ & $h_{3}$ & $\epsilon_{h_{3}}$ & $h_{4}$ & $\epsilon_{h_{4}}$ \\
\hline 0.00 & -6.51 & 1.29 & 211.91 & 5.50 & 0.069 & 0.053 & -0.062 & 0.016 \\
\hline 1.41 & -4.68 & 0.86 & 201.49 & 4.47 & 0.037 & 0.022 & -0.066 & 0.009 \\
\hline 3.76 & -7.94 & 1.04 & 188.69 & 3.30 & 0.000 & 0.015 & -0.053 & 0.009 \\
\hline 6.11 & -6.27 & 2.88 & 193.16 & 4.54 & -0.012 & 0.024 & -0.053 & 0.011 \\
\hline 8.46 & 1.79 & 2.63 & 198.46 & 4.27 & 0.005 & 0.027 & -0.049 & 0.011 \\
\hline 10.81 & -6.24 & 2.72 & 185.65 & 3.66 & -0.015 & 0.020 & -0.046 & 0.011 \\
\hline 13.16 & -1.07 & 4.47 & 195.26 & 6.04 & 0.083 & 0.029 & 0.025 & 0.024 \\
\hline 15.51 & 14.06 & 5.22 & 209.36 & 5.76 & -0.033 & 0.028 & 0.010 & 0.020 \\
\hline 17.86 & 6.49 & 6.04 & 180.82 & 7.07 & -0.043 & 0.033 & -0.059 & 0.016 \\
\hline 20.21 & -31.59 & 6.41 & 182.22 & 12.62 & 0.055 & 0.045 & 0.021 & 0.024 \\
\hline 22.56 & 30.06 & 12.08 & 219.63 & 11.81 & -0.031 & 0.037 & -0.030 & 0.026 \\
\hline 26.08 & -40.85 & 10.30 & 177.36 & 11.96 & -0.044 & 0.035 & -0.021 & 0.022 \\
\hline 30.78 & 5.47 & 10.77 & 194.77 & 12.27 & -0.036 & 0.037 & -0.034 & 0.024 \\
\hline 35.49 & -23.16 & 14.05 & 171.31 & 11.39 & 0.100 & 0.041 & 0.016 & 0.028 \\
\hline 41.36 & -76.04 & 21.58 & 233.45 & 21.95 & 0.020 & 0.050 & -0.004 & 0.032 \\
\hline
\end{tabular}

multi-object spectroscopy of globular clusters. Their results show good agreement with our $V$ and $\sigma$ profiles, as seen in their Figure 17. Previously, Proctor et al. (2005) determined the kinematics of NGC 821 using the Gemini Multi-Object Spectrograph (GMOS). As shown in Figure 2 of that paper, our $\sigma$ is in agreement with theirs, except that they find a lower dispersion at about 30". However, their data are of substantially lower S/N and they are also unable to determine higher-order moments.

Weijmans et al. (2009) provide a new analysis of the SAURON data and also include additional data at large radii. Their furthest radial point is at $110^{\prime \prime}$ (which they refer to as $4 R_{e}$ ), whereas our last point is at $90^{\prime \prime}$ (which we refer to as $2 R_{e}$ ). The comparison between the two kinematic sets is shown in Figure 6 from Weijmans et al. (2009). There is generally excellent agreement between the two sets of kinematics. Furthermore, their re-analysis of the SAURON central pointing shows $h_{4}$ values now more consistent with our numbers. The higherorder moments of the LOSVD are difficult to measure, and it is important to consider systematic difference in the analysis. The spectra from Weijmans et al. (2009) have lower S/N than our spectra, which could add to systematic difference. For this reason, our dynamical modeling does not include their kinematics, although we suspect that there will be little difference in the overall results. Thus, we can compare the constraints on the dark matter parameters, which would include systematic differences in the kinematic samples used.

Our results are consistent with Weijmans et al. (2009) and not consistent with either Romanowsky et al. (2003) or Coccato et al. (2009). The Weijmans et al. data are consistent with that of Coccato et al. The reason is simply that the Weijmans et al. dispersions have large uncertainties and that the Weijmans et al. dispersions are between our values and that of Coccato et al. The difference is not due to comparing dispersions measured from major axis radii compared to circular radii (as in Coccato et al.), since our dispersion profile is nearly flat. We compare with the Romanowsky et al. values and not to the values reported in Coccato. The differences between Coccato et al. and Romanowsky et al. are small enough not to impact our analysis or conclusions. The Coccato et al. data have slightly smaller uncertainties than Romanowsky et al., so the statistical differences between our dispersions and theirs are slightly larger.

Figure 3 shows the rms LOS velocity $\left(V^{2}+\sigma^{2}\right)^{1 / 2}$ compared to the planetary nebula results of Romanowsky et al. (2003).

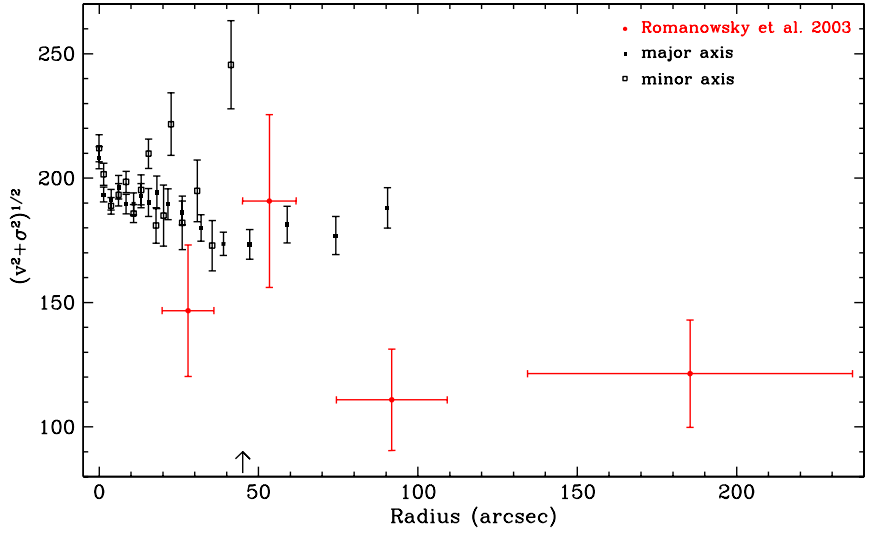

Figure 3. rms LOS velocity $\left(v^{2}+\sigma^{2}\right)^{1 / 2}$ in $\mathrm{km} \mathrm{s}^{-1}$ as a function of radius from our major axis data (filled squares) and minor axis data (open squares), compared to the data from planetary nebulae measurements (Romanowsky et al. 2003). We use $v$ and $\sigma$ as measure from a Gauss-Hermite fit; since we do not correct for the higher-order moments, these values approximate the actual second moment. The arrow indicates the adopted $R_{e}$ of the galaxy.

(A color version of this figure is available in the online journal.)

Our largest radii data show higher rms LOS velocities than the planetary nebulae, at about $3 \sigma$ for their two largest radii points. Thus, there appears to be a significant difference in the kinematics between the two samples.

\section{DYNAMICAL MODELS}

We use axisymmetric orbit-superposition models based on the method of Schwarzschild (1979). The surface brightness profile is converted to a luminosity density profile using an assumed inclination. We assume an edge-on inclination for this analysis, which is reasonable given NGC 821's large ellipticity of 0.40 (Cappellari et al. 2007). This luminosity density is converted to a mass density using a mass-to-light ratio $\left(M / L_{V}\right)$ that is constant over the galaxy. A spherically symmetric dark halo density profile is added to the stellar density and this total mass density gives the galaxy's gravitational potential. Next, individual stellar orbits are sampled in energy $(E)$, angular momentum $\left(L_{z}\right)$, and the third integral $\left(I_{3}\right)$, and these orbits are integrated in the specified potentials. The galaxy is divided spatially into cells both in real space and in projection, and the amount of time that an orbit spends in a cell represents the mass contributed by that orbit. The orbits are combined with nonnegative weights to find the best-fitted superposition to match the data LOSVDs from both HET and SAURON and the light profile. The model incorporates seeing by convolving the light distribution for every orbit with the appropriate point-spread function before comparing with the data. This process is repeated for different dark halo density profiles and $M / L_{V}$ values to find the halo potential that best fits the data, as determined by $\chi^{2}$ (described in Section 5).

The SAURON data are described in Emsellem et al. (2004). We reconstruct a full LOSVD from their reported moments and rebin them to match our model bins. The Pinkney et al. (2003) data shown in Figure 2 are not used in the models.

To reduce computational time, an orbit library is calculated for a given input dark halo plus stars with a mass-to-light ratio $(M / L)$ of one. The velocities are then scaled accordingly given the $M / L$ before matching the data. The numbers reported are the actual density parameters, including this $M / L$ factor, which gives the somewhat irregular parameter space grids (as seen in Figure 5). 
Table 3

$V$-band Luminosity Density

\begin{tabular}{cc}
\hline \hline Radius $\left(^{\prime \prime}\right)$ & $L_{\odot} \mathrm{pc}^{-3}$ \\
\hline $2.300 \mathrm{E}-02$ & $8.275 \mathrm{E}+03$ \\
$2.533 \mathrm{E}-02$ & $7.205 \mathrm{E}+03$ \\
$2.790 \mathrm{E}-02$ & $6.283 \mathrm{E}+03$ \\
$3.073 \mathrm{E}-02$ & $5.485 \mathrm{E}+03$ \\
$3.385 \mathrm{E}-02$ & $4.793 \mathrm{E}+03$ \\
$3.728 \mathrm{E}-02$ & $4.192 \mathrm{E}+03$ \\
$4.107 \mathrm{E}-02$ & $3.668 \mathrm{E}+03$ \\
$4.523 \mathrm{E}-02$ & $3.212 \mathrm{E}+03$ \\
$4.982 \mathrm{E}-02$ & $2.813 \mathrm{E}+03$ \\
$5.487 \mathrm{E}-02$ & $2.464 \mathrm{E}+03$ \\
$\ldots$ & $\cdots$ \\
\hline
\end{tabular}

(This table is available in its entirety in a machinereadable form in the online journal. A portion is shown here for guidance regarding its form and content.)

We use the orbital weight fitting of Gebhardt et al. (2000, 2003) with the orbit library sampling of Thomas et al. (2004, 2005). Our models differ from others (e.g., Cretton et al. 1999 ) in that we use maximum entropy (Richstone \& Tremaine 1988) and we utilize the full LOSVD, rather than its moments. Thomas et al. $(2004,2005)$ show the ability of our orbit libraries to recover dark halo profiles from mock elliptical galaxy data. Therefore, these models should accurately measure the properties of NGC 821 given the caveats that we assume an axisymmetric galaxy and spherical halo.

The orbits are computed in 4 angular bins and 15 radial bins from 0.3 to $300^{\prime \prime}$. These bins are similar in size to the HET data extraction bins in the radial direction, though in the angular direction they span about $20^{\circ}$. We specify the galaxy potential and the forces on a grid that is 4 times finer. Our libraries have approximately 10,000 total orbits.

To calculate our galaxy potential, we use a composite surface brightness profile. Within 0.3, we use the profile from Lauer et al. (2005) as compiled in Pinkney et al. (2003) based on Hubble Space Telescope (HST) WFPC2 images in F555W. Outside of 0.3 , we use a composite profile from HST PC F555W and the McDonald Observatory $0.8 \mathrm{~m}$ telescope in $V$ (D. Fisher 2005, private communication). The surface brightness deprojection is based on a nonparametric estimate of the density using smoothing splines (see Gebhardt et al. 1996). The luminosity density is given in Table 3.

\subsection{NFW Halo}

We use the Navarro-Frenk-White (NFW; Navarro et al. 1996) dark halo density profile, given as

$$
\rho(r)=\frac{\rho_{\text {crit }} \delta_{c}}{\left(r / r_{s}\right)\left(1+r / r_{s}\right)^{2}},
$$

where $r_{s}$ is the scale radius of the halo, $\rho_{\text {crit }}=3 H^{2} / 8 \pi G$ is the critical density, and $\delta_{c}$ is the characteristic overdensity. We use $H=70 \mathrm{~km} \mathrm{~s}^{-1} \mathrm{Mpc}^{-1}$. Throughout this paper, we refer to $\rho_{\text {crit }} \delta_{c}$ as the scale density. We use two independent parameters to define the NFW halo in our models: the scale density and the scale radius. The scale density can also be written in terms of a concentration parameter $c$ by

$$
\delta_{c}=\frac{\Delta_{\mathrm{vir}}}{3} \frac{c^{3}}{\ln (1+c)-c /(1+c)} .
$$

The virial overdensity $\Delta_{\text {vir }}$ varies with redshift and cosmological model and we use a value of $\Delta_{\text {vir }}=101$. Although we vary both the concentration (density) and scale radius, there is a known correlation between them (Navarro et al. 1996). This relation as given in Bullock et al. (2001) is

$$
c \simeq 9\left(\frac{M_{\mathrm{vir}}}{1.5 \times 10^{13} h^{-1} M_{\odot}}\right)^{-0.13}
$$

and can be written in the form

$$
r_{s}^{3}=\left(\frac{c}{9}\right)^{-1 / 0.13}\left(\Delta_{\text {vir }} \frac{4 \pi}{3} \rho_{\text {crit }} c^{3}\right)^{-1}\left(1.5 \times 10^{13} h^{-1} M_{\odot}\right)
$$

\subsection{Power-law Halo}

The best-fitted NFW halo profiles have a break radius beyond the extent of our modeling and therefore look like a power law over the extent of our models (see Section 5.1). We therefore tried a simple power-law profile as well. We used power-law density profiles of the form

$$
\rho(r)=\rho_{o}\left(\frac{r}{r_{o}}\right)^{-n},
$$

where $n$ is the power-law slope, $\rho_{o}$ is the characteristic density, and $r_{o}$ is the characteristic radius such that $\rho\left(r=r_{o}\right)=\rho_{o}$. We use $r_{o}=0.3=34 \mathrm{pc}$ because it is the inner-most radial point calculated in the models.

\section{RESULTS}

The best-fitted model is determined by comparing the $\chi^{2}$ between the model and data LOSVDs, with the uncertainty of the data determined from the $68 \%$ confidence band. Example LOSVDs are shown in Figure 4 for several radial bins. The measure of the reduced $\chi^{2}$ is not straightforward because determining the number of degrees of freedom is uncertain. The number of independent observables is roughly the number of radial data bins times the number of LOSVD bins at each radius $(69 \times 13=897$ in this case); however, the LOSVD bins are correlated and thus the effective number of data points is less than this value. The best-fitted model has a $\chi^{2}$ of around 2200 , and with 897 data points this provides a large reduced $\chi^{2}$. Typical values of the reduced $\chi^{2}$ for the orbit-based models are around 0.5 (see Gebhardt et al. 2003), so the value reported here is not typical. The main driver for the large $\chi^{2}$ is the minor axis data-removing this data gives a reduced $\chi^{2}$ below one. Furthermore, the results on the parameters do not change significantly. Regardless, the change in $\chi^{2}$ between different models remains a valid statistic to determine confidence levels of the fits. For example, a change in $\chi^{2}$ of 2.3 corresponds to the $68.3 \%$ confidence level because we marginalize over $M / L$ and thus have two parameters describing the halo.

Because of computational limits, we first calculate models using a coarse grid of $M / L$. The $\chi^{2}$ values are then fit with the IDL quadratic interpolation routine, and those models with the lowest minimum $\chi^{2}$ are modeled with a finer $M / L$ interval.

\subsection{NFW Halo}

We use models with scale radius from 1 to $2000 \mathrm{kpc}$ and scale density from 0.05 to $3.0 \times 10^{-5} M_{\odot} \mathrm{pc}^{-3}$, corresponding to a range in $c$ of approximately $0.75-23$ and $M / L_{V}$ from 1.0 to 9.0. Figures 5 and 6 show the resulting $\chi^{2}$ as a function of 


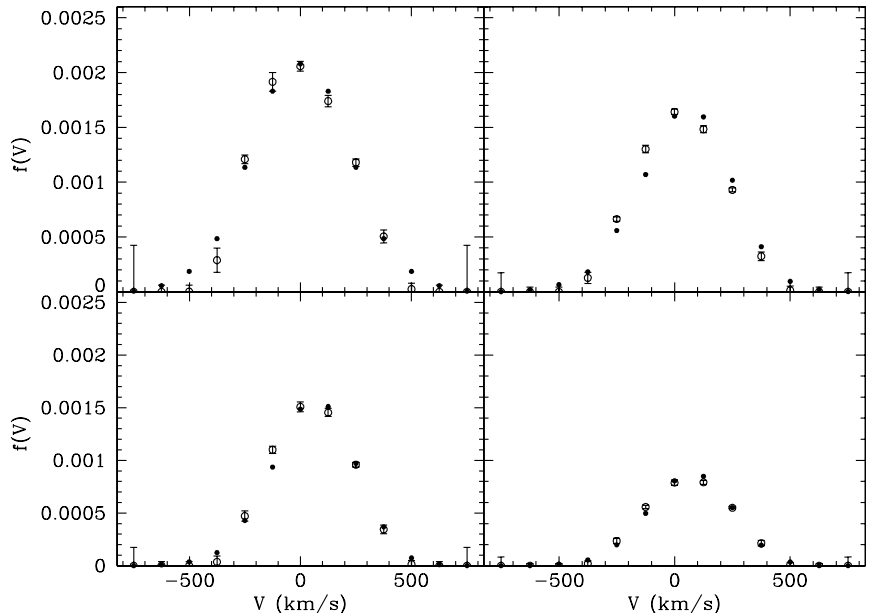

Figure 4. Match of data and no dark halo model LOSVDs for the central four radial bins along the major axis $(r=0.00,1.41,3.76,6.11 \mathrm{arcsec})$. The open circles are the data values with error bars and the closed circles are the model values. The area is normalized to the total light in that bin.

halo scale radius and scale density. The points represent actual modeled values, and the $M / L_{V}$ that gives the lowest $\chi^{2}$ is used at each point. The dashed line in Figure 5 indicates the expected correlation of concentration and scale radius as described in Section 4.1. This relation has a scatter of $\Delta \log r_{s}=0.36$ (Bullock et al. 2001). Our data show a degeneracy between scale radius and scale density that is similar to, though slightly tilted from, the correlation.

$\chi^{2}$ is a function of three variables: stellar $M / L$, dark halo scale radius, and dark halo normalization. Due to computer re-

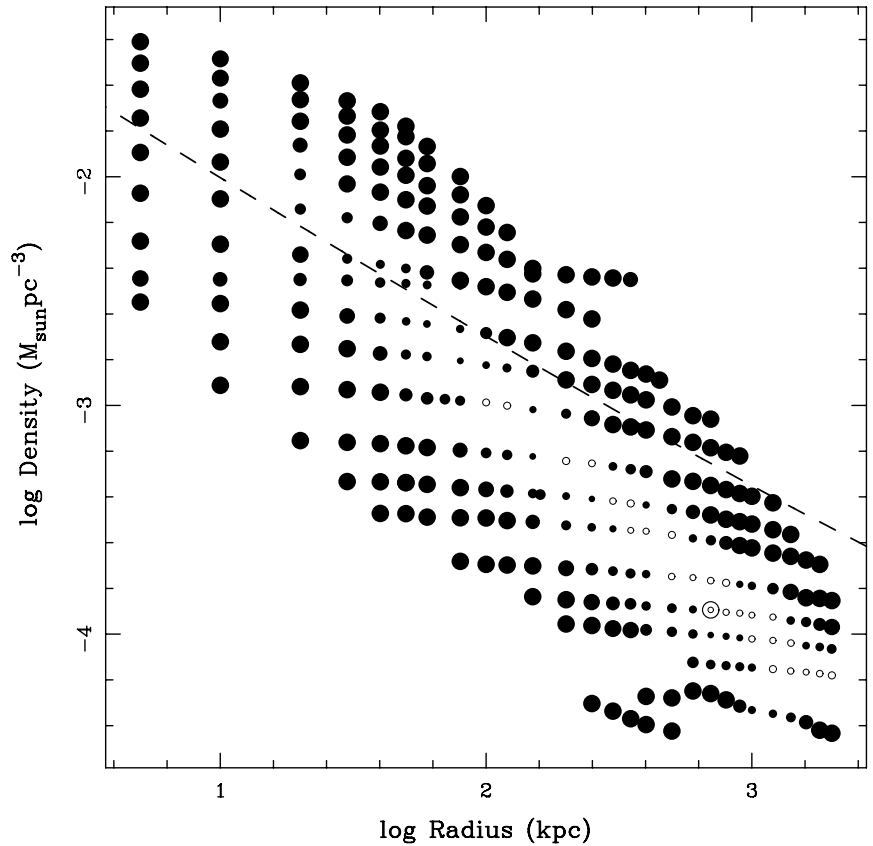

Figure 5. Scale radius $\left(r_{s}\right)$ and scale density $\left(\rho_{\text {crit }} \delta_{c}\right) \chi^{2}$ grid for NFW halo density profiles. Each point represents a model, and the size of the point reflects the value of $\Delta \chi^{2}$ for the best-fitted $M / L_{V}$ value. Models with $\Delta \chi^{2}$ less than $6 \sigma$ from the minimum value are plotted with open circles. The ringed point indicates the model with the lowest value of $\chi^{2}$. The dashed line shows the expected NFW parameter relation (see Section 4.1)

sources, we do not provide a uniformally sampled grid of the three variables for the $\chi^{2}$. Because of this, it is difficult to

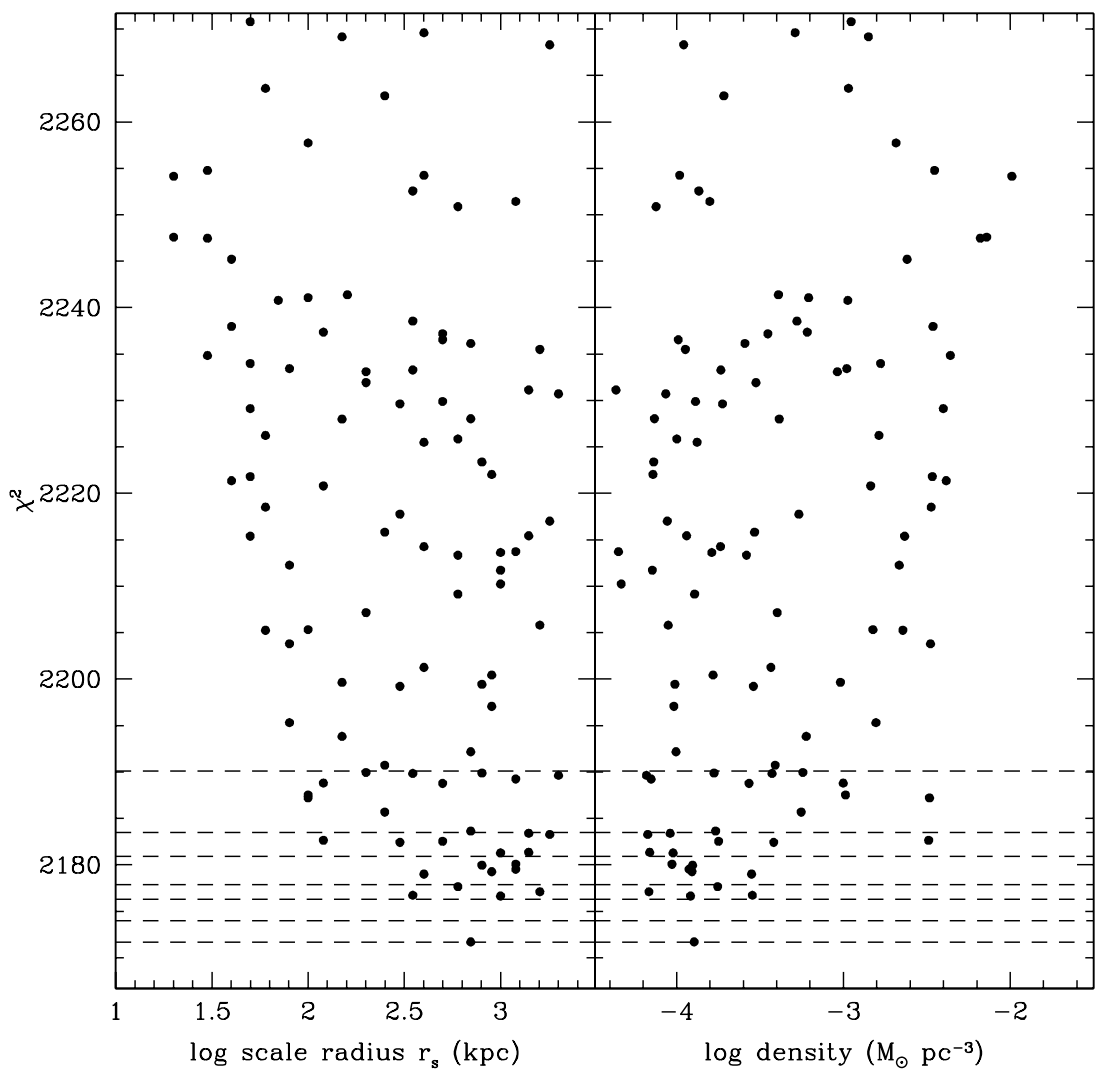

Figure 6. $\chi^{2}$ as a function of scale radius and scale density $\left(\rho_{\text {crit }} \delta_{c}\right)$ for NFW halo density profiles with best-fitted $M / L_{V}$. The points represent actual modeled values. The dashed lines refer to $\Delta \chi^{2}=2.3,4.61,6.17,9.21,11.8,18.4$, corresponding to 2 degrees of freedom confidence levels of $63.8 \%, 90 \%, 95.4 \%, 99 \%, 99.73 \%$, and $99.99 \%$. 
Table 4

Best-fitted Halo Model Results

\begin{tabular}{|c|c|c|c|c|c|c|c|c|c|c|}
\hline Halo & $\begin{array}{l}\chi^{2} \\
(2)\end{array}$ & $\begin{array}{c}M / L_{V} \\
(M / L)_{\odot} \\
(3)\end{array}$ & $\begin{array}{c}r_{s} \\
(\mathrm{kpc}) \\
(4)\end{array}$ & $\begin{array}{c}\rho \\
\left(M_{\odot} \mathrm{pc}^{-3}\right) \\
(5)\end{array}$ & (6) & $\begin{array}{c}M_{\text {vir }} \\
10^{17} M_{\odot} \\
(7)\end{array}$ & $\begin{array}{c}n \\
(8) \\
\end{array}$ & $\begin{array}{c}M_{\text {tot }} \\
10^{11} M_{\odot} \\
(9)\end{array}$ & $\begin{array}{c}M_{\text {stars }} \\
10^{11} M_{\odot} \\
(10)\end{array}$ & $\begin{array}{c}M_{\text {halo }} \\
10^{11} M_{\odot}\end{array}$ \\
\hline None & 2527.30 & $7.25 \pm 0.05$ & & & & $\ldots$ & & $1.20 \pm 0.01$ & $1.20 \pm 0.01$ & 0.00 \\
\hline NFW & 2171.70 & $6.19 \pm 0.09$ & $700_{-300}^{+500}$ & $1.28_{-0.5}^{+0.8} \times 10^{-4}$ & $2.45_{-0.53}^{+0.65}$ & $4.76_{-4.0}^{+26}$ & $\cdots$ & $1.78 \pm 0.15$ & $1.03 \pm 0.03$ & $0.75 \pm 0.15$ \\
\hline Power law & 2077.05 & $6.25 \pm 0.07$ & $\ldots$ & $0.025_{-0.009}^{+0.025}$ & $\ldots$ & $\ldots$ & $0.1_{-0.08}^{+0.1}$ & $2.01 \pm 0.15$ & $1.04 \pm 0.02$ & $0.97 \pm 0.15$ \\
\hline
\end{tabular}

Notes. (1) Dark halo density profile. (2) $\chi^{2}$ of best-fitted model. (3) Stellar $M / L_{V}$ of best-fitted model. (4) Scale radius of best-fitted model. (5) Scale density $\rho_{\text {crit }} \delta_{c}$ for NFW, characteristic density $\rho_{o}$ for power law. (6) NFW concentration parameter determined from scale density. (7) Virial mass determined from NFW concentration parameter. (8) Power-law index. (9) Total mass within 100". (10) Mass of stars within 100". (11) Mass of dark halo within 100".
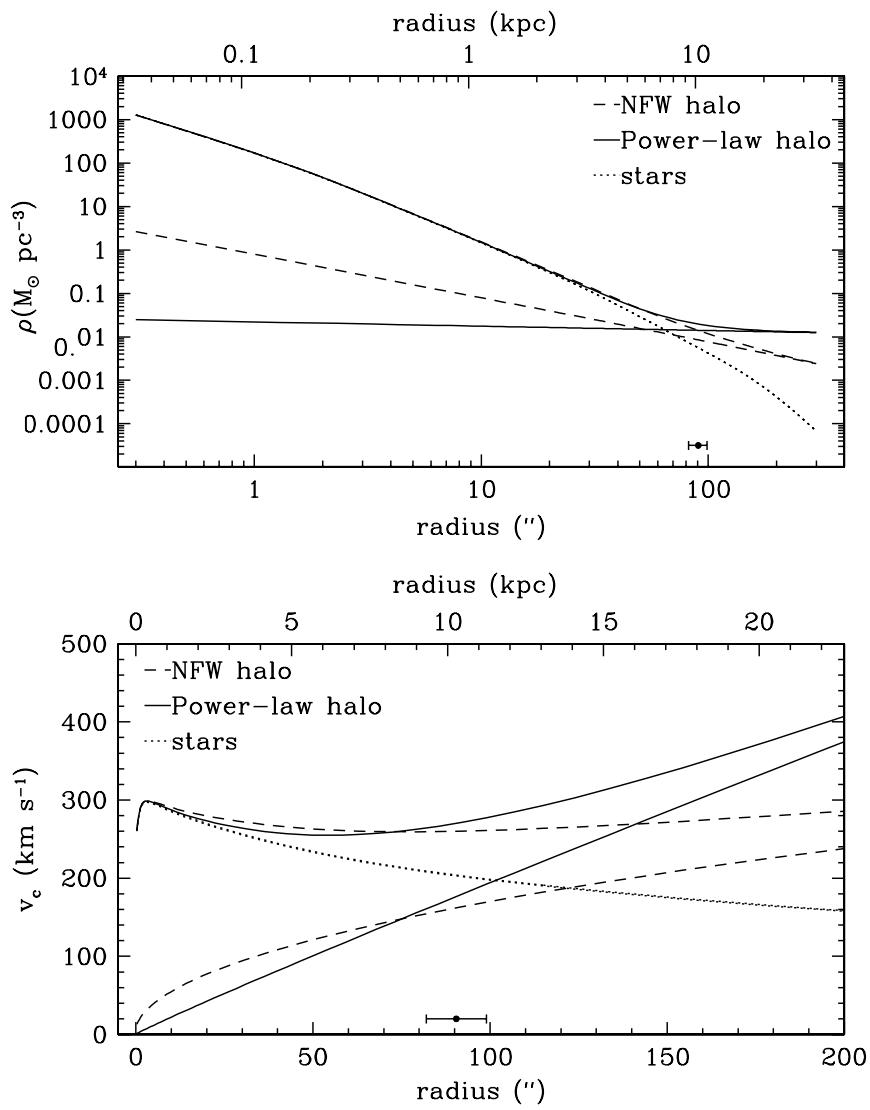

Figure 7. Density (top) and circular velocity (bottom) as a function of radius for the best-fitted NFW (dashed lines) and power-law (solid lines) dark halos. In each case, the bottom line is the dark halo alone and the top line is the total mass (halo plus stars). The data point shows the radius of the most extended bin of our kinematic data

produce reliable contours for any two of the parameters. Figure 5 thus shows only the location of the points (with size related to $\chi^{2}$ ). We do not estimate uncertainties from the contours directly, but instead rely on plotting $\chi^{2}$ versus each of the parameters, including all values for the other two parameters. Figure 6 shows $\chi^{2}$ versus scale radius and density. Uncertainties come from the envelope of these one-dimensional plots. Since we have explored neither a regular grid nor a full set of variables (e.g., black hole mass, inclination, and change in the stellar $M / L$ with radius), the uncertainties should only be used in a comparative sense with the models that we have tried. A full exploration of the uncertainties will come as computer resources improve.

We find that the best-fitted NFW dark halo density profile has scale radius $700_{-300}^{+500} \mathrm{kpc}$ and scale density $1.28_{-0.5}^{+0.8} \times$ $10^{-4} M_{\odot} \mathrm{pc}^{-3}$, corresponding to a $c$ of 2.45 . The no-halo model

is ruled out with a change in $\chi^{2}$ of 356 (greater than $99 \%$ confidence level) from the best-fitted NFW halo. Table 4 shows the $\chi^{2}$ values and halo parameters of the best-fitted halo model and model with no dark halo. We do not attach significance to this density, radius, and concentration. They are clearly outside the expected range for a galaxy and merely indicate that the NFW profile is not reasonable. The halo needs more mass at large radii to fit the data. The best-fitted NFW halo density profile is shown in Figure 7. The scale radius is well beyond the radial extent of our modeling and is indicative of the need for a near power-law profile over the extent of our models.

Since dynamical modeling directly measures mass (as opposed to dark halo parameters), the enclosed mass provides a more robust estimate and is likely not subject to the specific parameterization of the dark halo. Figure 8 shows the mass enclosed within the extent of our kinematic data as a function of $\chi^{2}$. The best-fitted total enclosed mass is $1.78 \pm 0.15 \times 10^{11} M_{\odot}$, divided into $1.03 \pm 0.03 \times 10^{11} M_{\odot}$ in stars and $0.75 \pm 0.15 \times$ $10^{11} M_{\odot}$ in dark matter. At $1 R_{e}$, the ratio of dark matter to total matter is 0.19 . The best-fitted NFW halo circular velocity profile is shown in Figure 7.

Figures 9 and 10 show the internal moments $\sigma_{r}, \sigma_{\theta}$, and $\sigma_{\phi}$ and ratio of radial to tangential dispersion along the major and minor axes for the model with no dark halo and the best-fitted NFW halo model. The model without a dark halo shows radial anisotropy at small radii and tangential anisotropy at large radii along the major axis. Tangential anisotropy at large radii in a model with no dark halo could be an indication of the need for a dark halo because the observations largely constrain only $\sigma_{\phi}$ (for an edge-on configuration), so both $\sigma_{r}$ and $\sigma_{\theta}$ may be artificially decreased to create a smaller total $\sigma$ that can be fit without a dark halo. Along the minor axis, the contribution in the $\theta$ and $\phi$ directions is roughly equal, as is expected for an axisymmetric model. Overall the minor axis shows tangential anisotropy over the entire range of our data. The NFW model is more isotropic than the model with no halo.

Although the models fit the full nonparametric velocity profile of both the HET data and SAURON data, in Figure 11 we plot the first four Gauss-Hermite moments for our HET data and the best-fitted halo models. The models differ most at intermediate to large radii and do not appear to be driven by any one single parameter or radius in particular.

\subsection{Power-law Halo}

We run models with a range of slopes $n$ from 0.0 to 1.1 , densities $\rho_{o}$ from 0.0015 to $26 M_{\odot} \mathrm{pc}^{-3}$, and $M / L_{V}$ from 3.5 to 8.0. The resulting $\chi^{2}$ grid is shown in Figure 12 and as a function of $n$ and $\rho_{o}$ in Figure 13. The best-fitted halo model has a slope $0.1_{-0.08}^{+0.1}$ and a characteristic density $\rho_{o}=0.025_{-0.009}^{+0.025} M_{\odot} \mathrm{pc}^{-3}$. This halo is a better fit to the data than the best NFW halo, 


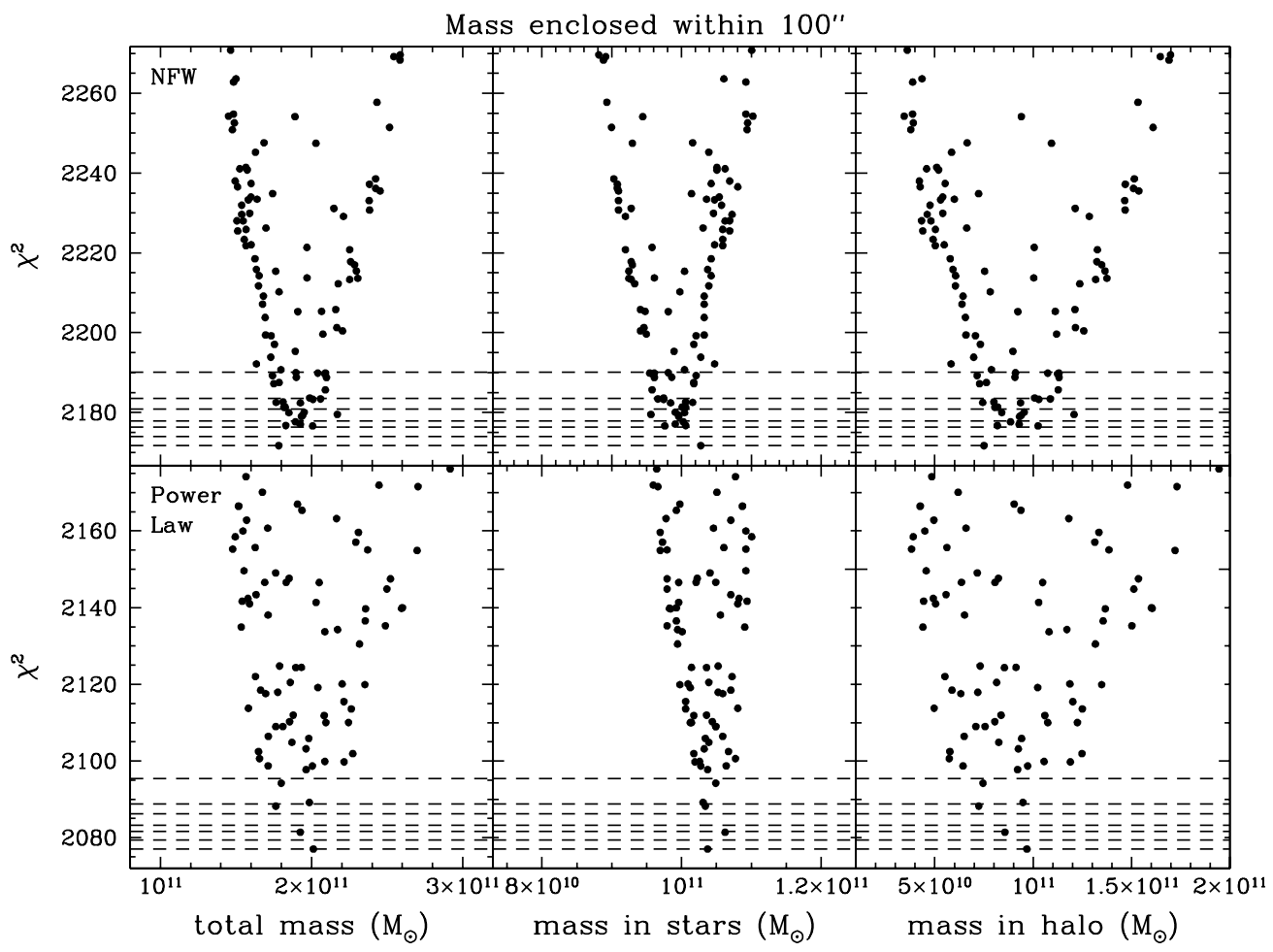

Figure 8. Enclosed mass within the radial extent of our kinematic data, $100^{\prime \prime}$, as a function of $\chi^{2}$ for both the NFW halo density profiles (top) and power-law halo density profiles (bottom) with best-fitted $M / L_{V}$. The dashed lines refer to $\Delta \chi^{2}=2.3,4.61,6.17,9.21,11.8,18.4$, corresponding to 2 degrees of freedom confidence levels of $63.8 \%, 90 \%, 95.4 \%, 99 \%, 99.73 \%$, and $99.99 \%$.
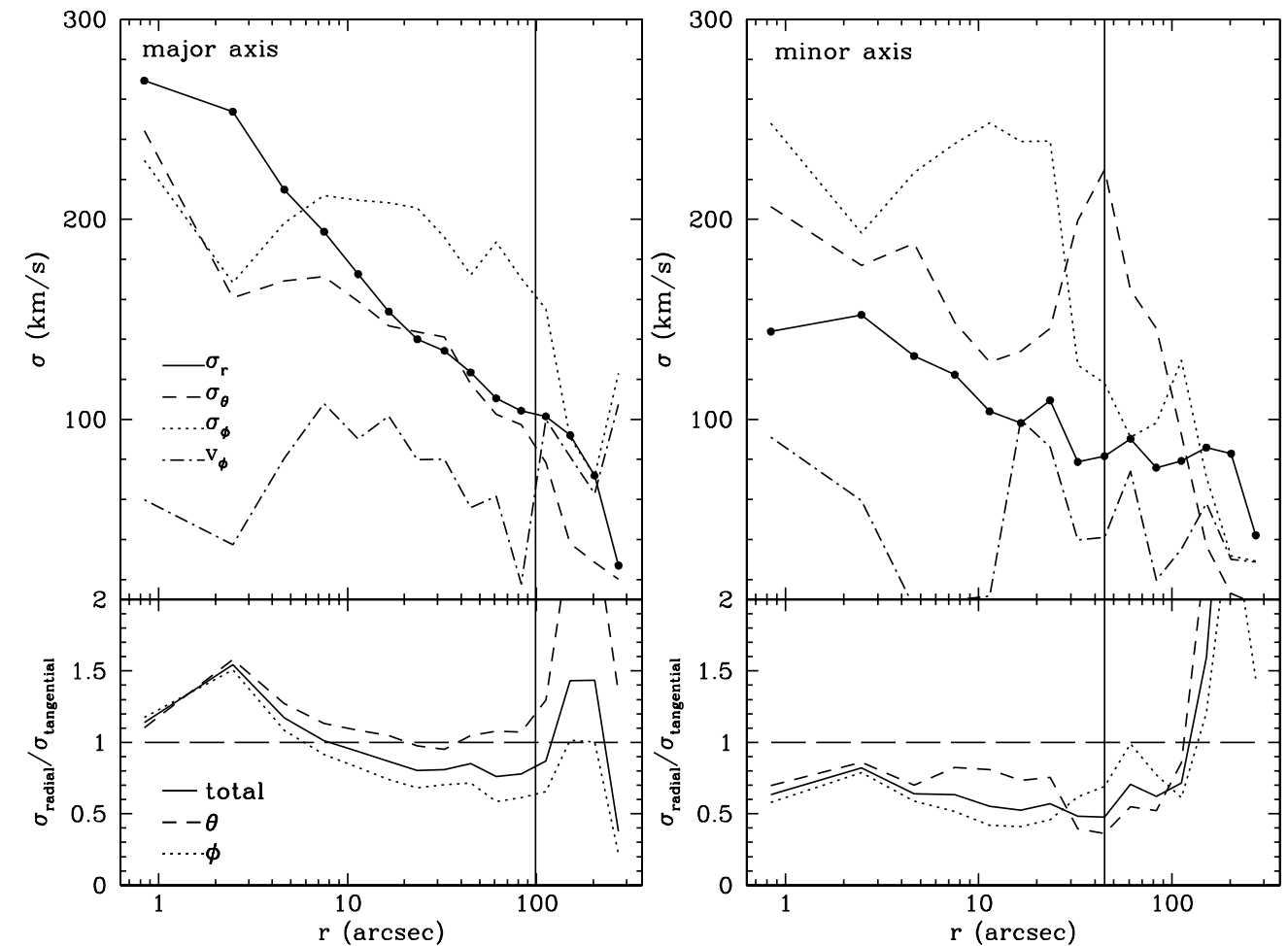

Figure 9. Internal moments $\sigma_{r}, \sigma_{\theta}$, and $\sigma_{\phi}$ (top) and the ratio of radial to tangential dispersion (bottom) along the major axis (left) and minor axis (right) for the model with no dark halo. Note that $\sigma_{\phi}$ includes both random and ordered motions, which are shown (dot-dashed line) and are small. The vertical line shows the limit of our kinematic data; results beyond this radius are not reliable.

with a $\Delta \chi^{2}=95$ (see Table 4). This power-law slope is significantly more shallow than the 1.0 slope of an NFW profile. A comparison of the best-fitted halo density and circular velocity profiles is shown in Figure 7.
The best-fitted total enclosed mass is $2.01 \pm 0.15 \times 10^{11} M_{\odot}$, divided into $1.04 \pm 0.02 \times 10^{11} M_{\odot}$ in stars and $0.97 \pm 0.15 \times$ $10^{11} M_{\odot}$ in dark matter (see Figure 8). At $1 R_{e}$, the ratio of dark matter to total matter is 0.13 . The internal moments $\sigma_{r}, \sigma_{\theta}$, and 

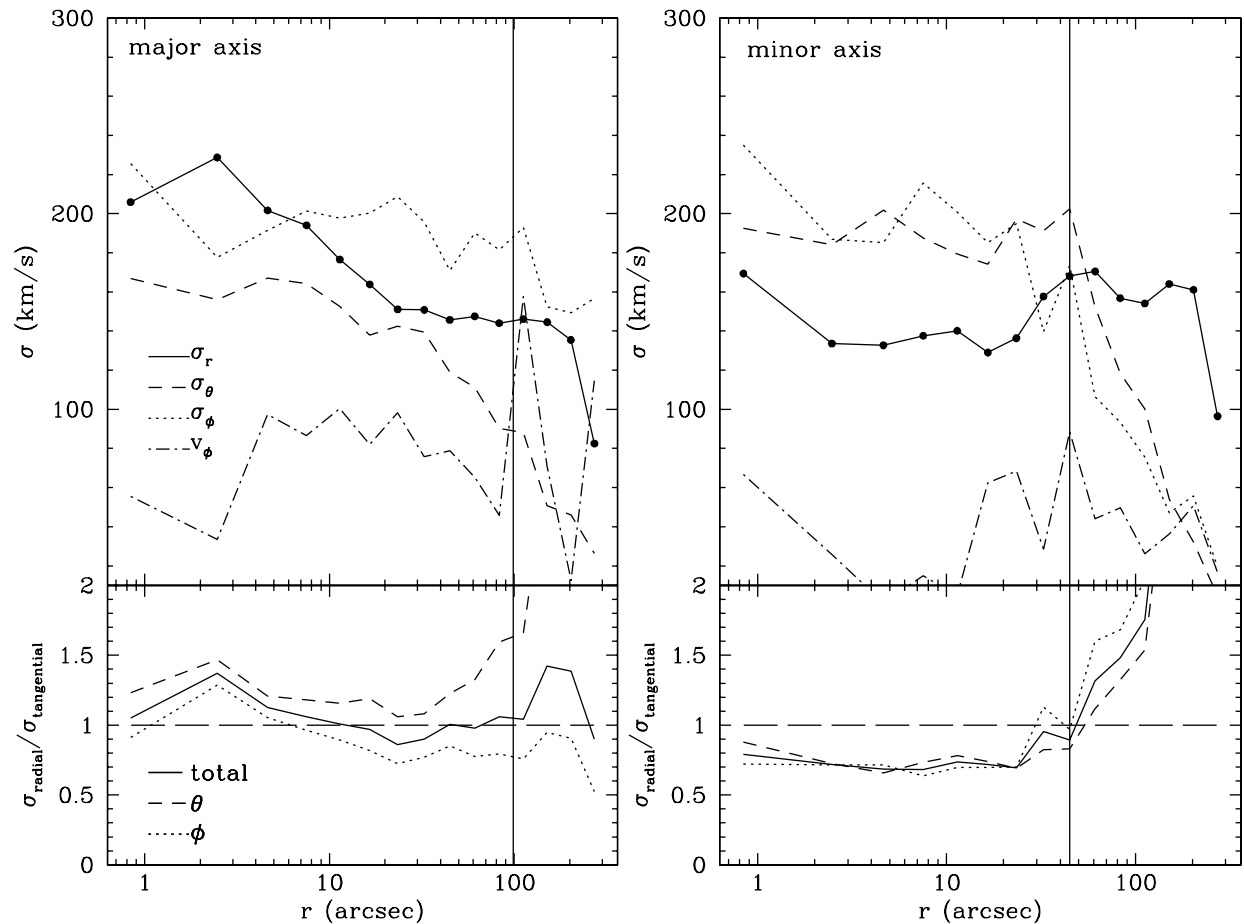

Figure 10. Internal moments $\sigma_{r}, \sigma_{\theta}$, and $\sigma_{\phi}$ (top) and the ratio of radial to tangential dispersion (bottom) along the major axis (left) and minor axis (right) for the model with the best-fitted NFW halo. Note that $\sigma_{\phi}$ includes both random and ordered motions, which are shown (dot-dashed line) and are small. The vertical line shows the limit of our kinematic data; results beyond this radius are not reliable.

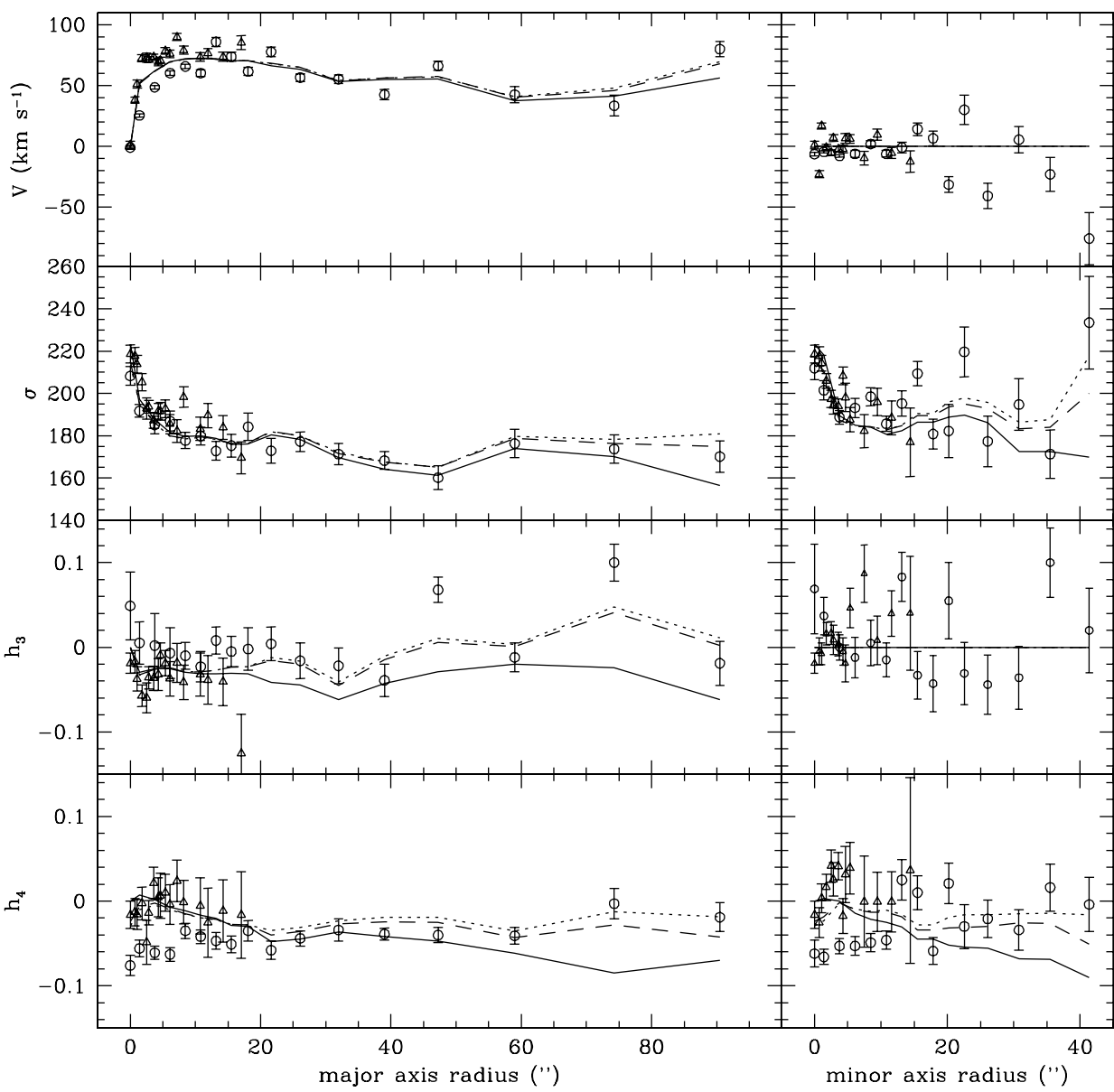

Figure 11. Gauss-Hermite moments (mean velocity $V$, velocity dispersion $\sigma$, asymmetric deviations from Gaussian (skewness) $h_{3}$, and symmetric deviations from Gaussian (kurtosis) $h_{4}$ ) of the LOSVDs for our HET data and the best-fitted halo models along the major axis (left panel) and minor axis (right panel). The HET data are shown with open circles, SAURON data along the axes with open triangles, no dark halo model with solid lines, best-fitted NFW halo with dashed lines, and best-fitted power-law halo with dotted lines. The model fits the full LOSVD of the HET data and all of the SAURON data. 


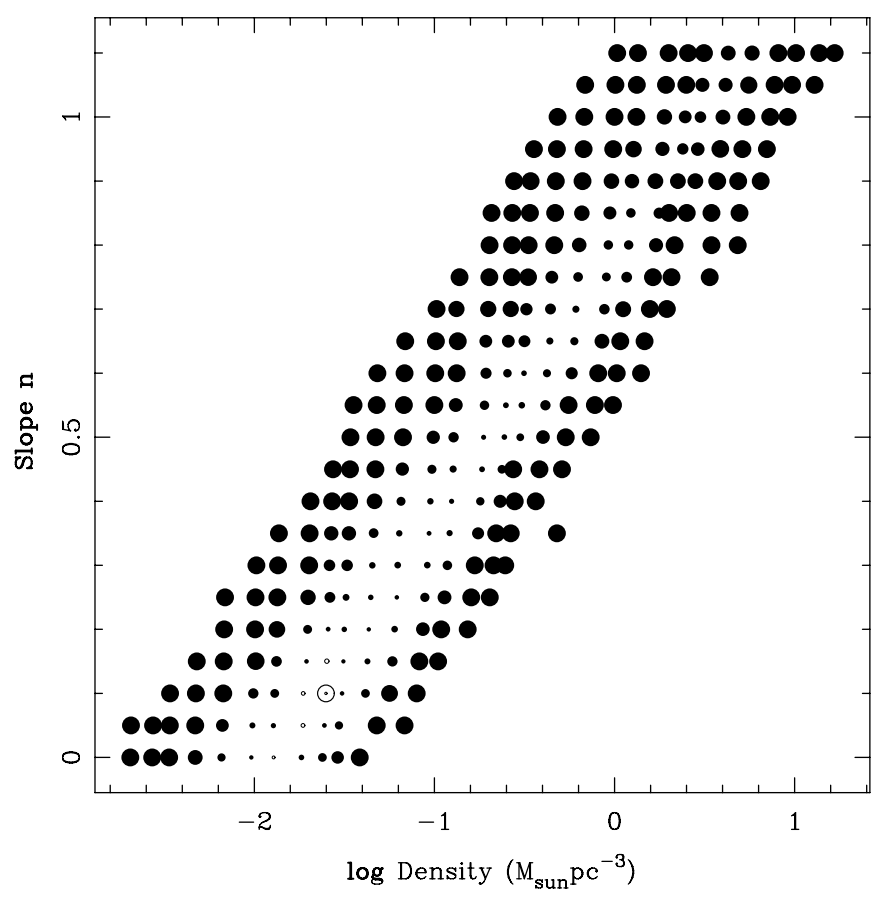

Figure 12. Power-law slope $n$ and density $\rho_{o} \chi^{2}$ grid for power-law halo density profiles. Each point represents a model, and the size of the point reflects the value of $\Delta \chi^{2}$ for the best-fitted $M / L_{V}$ value. Models with $\Delta \chi^{2}$ less than $6 \sigma$ from the minimum value are plotted with open circles. The ringed point indicates the model with the lowest value of $\chi^{2}$.

$\sigma_{\phi}$ and ratio of radial to tangential dispersion along the major axis are shown in Figure 14 and are roughly consistent with those of the best-fitted NFW halo.
Figure 11 shows the first four Gauss-Hermite moments for our HET data and the best-fitted halo models. Note that the models fit the full nonparametric velocity profile of both the HET data and SAURON data.

\subsection{Model Tests}

In order to learn which aspect of the data is driving the results, we have performed several tests. Using an abbreviated grid of about one third of the halos in the full run and a coarse spacing in $M / L_{V}$, we have re-run the models with various subsets of the data. First, to address any concerns over the scattered minor axis data we have run the test models using only the HET major axis data and SAURON data. The results are the same as the full data set; there is a clear need for a dark halo, and the best-fitted NFW halo is not as good a fit as the power-law halo. Second, we removed the two points on the major axis with extreme $h_{3}$ values (at about $47^{\prime \prime}$ and $74^{\prime \prime}$ ) since Figure 11 may lead one to believe that they are driving the fits. Again the results are the same as with the full data set. And third, we do a test run using only data below $0.5 R_{e}$. In this case, there is essentially no difference in $\chi^{2}$ between the three best-fitted models (no dark halo, NFW halo, and power-law halo), and the best-fitted halos are quite different than those from the full data results. These tests indicate that it is the large-radii data as a whole that is driving the model fits. To further demonstrate this, Figure 15 shows the $\Delta \chi^{2}$ between the model with no halo and the best-fitted halo in each bin. The bins at large radii show the greatest change in $\chi^{2}$, again indicating that the large-radii data are the major factor in the fits.

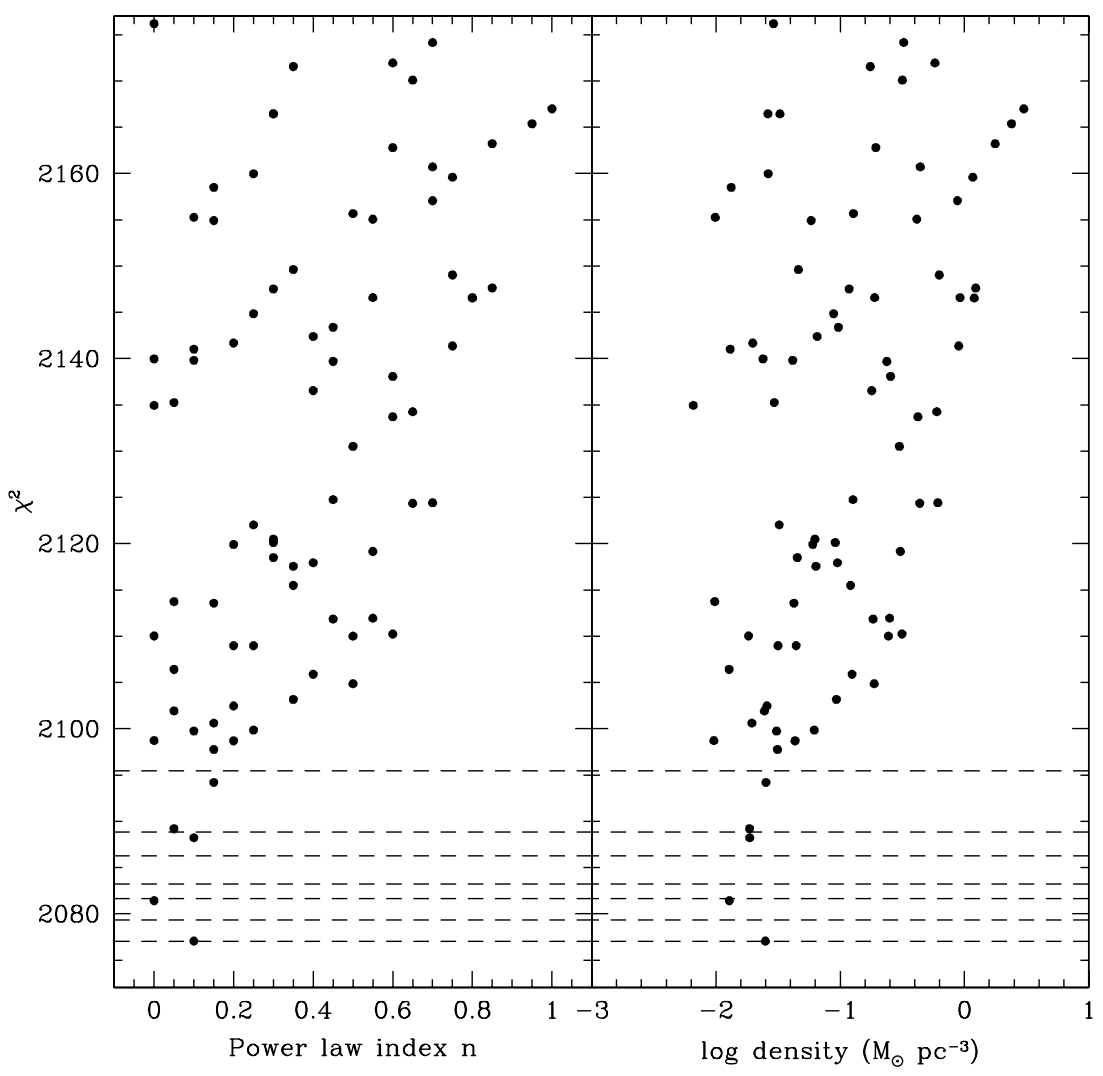

Figure 13. $\chi^{2}$ as a function of power-law index $n$ and scale density $\rho_{o}$ for power-law halo density profiles with best-fitted $M / L_{V}$. The points represent actual modeled values. The dashed lines refer to $\Delta \chi^{2}=2.3,4.61,6.17,9.21,11.8,18.4$, corresponding to 2 degrees of freedom confidence levels of $63.8 \%, 90 \%, 95.4 \%, 99 \%$, $99.73 \%$, and $99.99 \%$. 

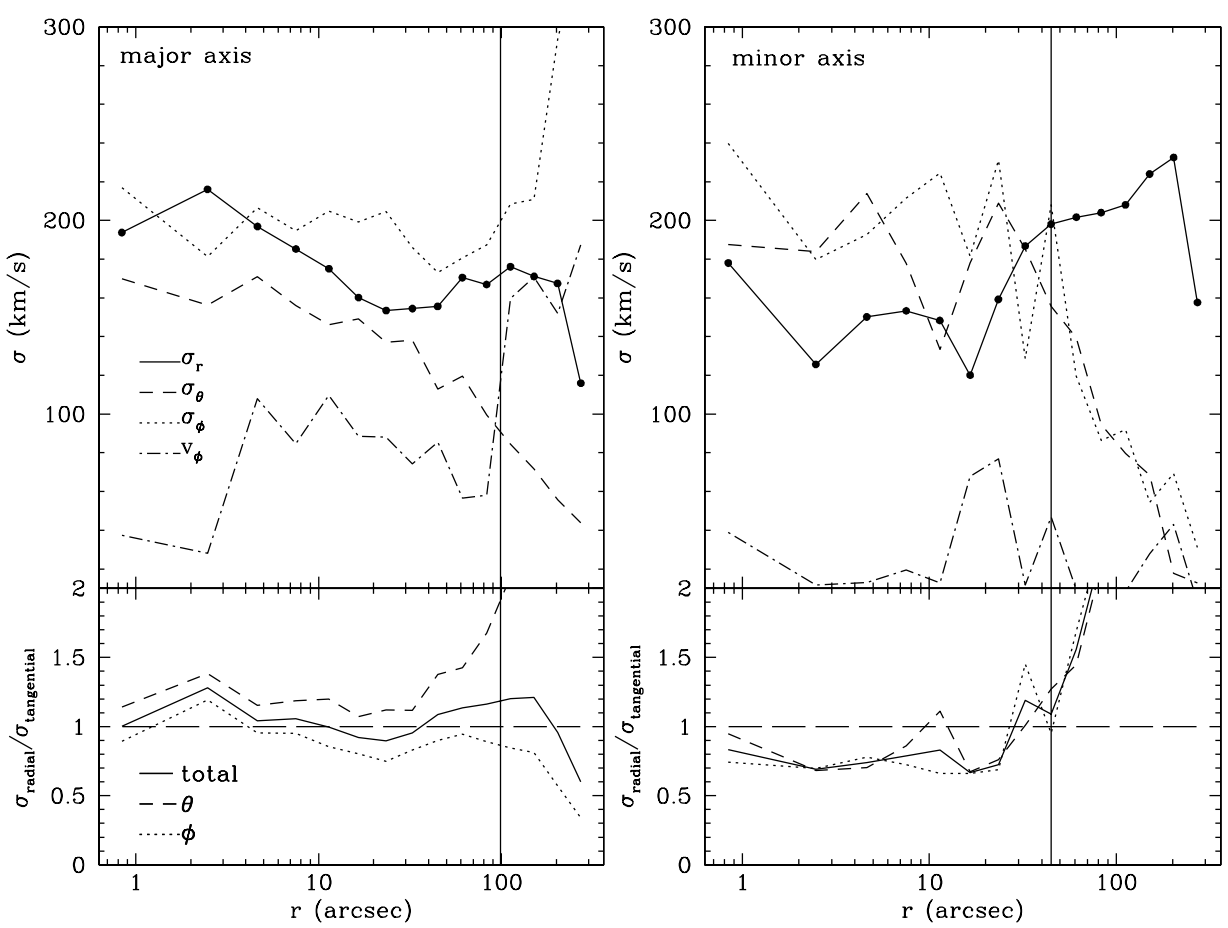

Figure 14. As in Figure 9, but for the best-fitted power-law model.

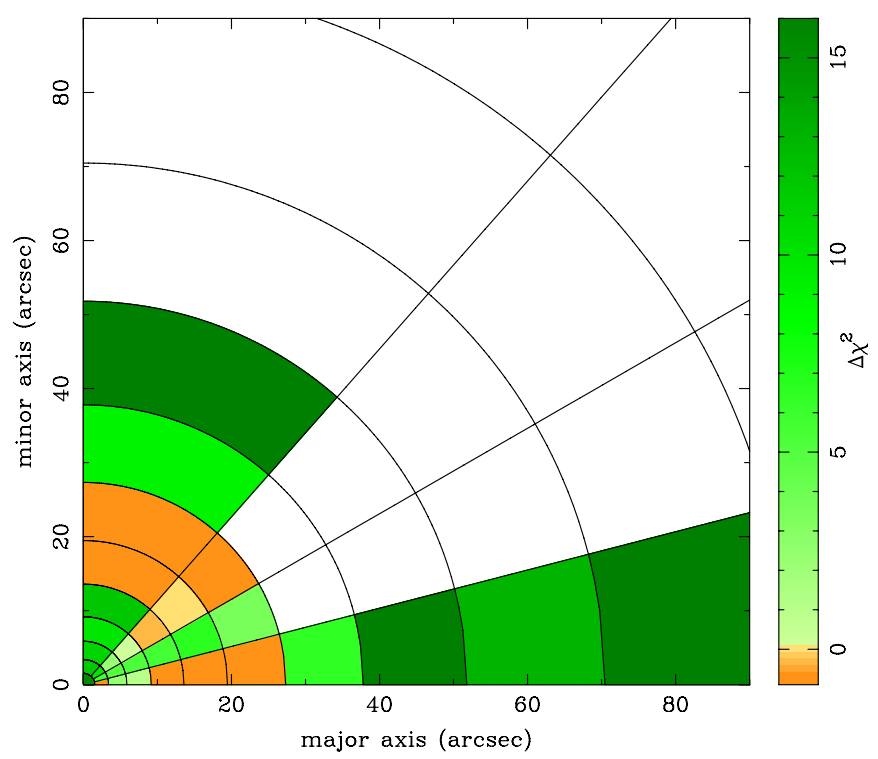

Figure 15. Difference in $\chi^{2}$ between the LOSVDs of the model with no dark halo and the best-fitted power-law halo model averaged in each spatial bin. Green indicates that the no-halo model has a larger $\chi^{2}$ than the power-law model and therefore the power law is a better fit, while orange indicates that the power-law model has a larger $\chi^{2}$ than the no-halo model and therefore the no-halo model is a better fit.

(A color version of this figure is available in the online journal.)

\subsection{Comparisons to Other Studies}

Gebhardt et al. (2003) model the central region of NGC 821 and find that it is radially anisotropic within a few arcseconds and isotropic to slightly tangentially anisotropic at larger radii. Given the difference in spatial resolution, this roughly agrees with our result. Cappellari et al. (2007) find that within about 20" N821 is radially anisotropic overall, along the major and minor axes and between. They find that the velocity ellipsoids are circular in the center and become more radial with increasing radius, in conflict with our results, but they do not include a dark halo which could change their results. Thomas et al. (2007), using similar modeling as we use, find that early-type galaxies in the Coma cluster are radial compared to the $\theta$ direction overall radii along the major axis, agreeing with our result, and vary from galaxy to galaxy in the $\phi$ component. The merger simulations of Dekel et al. (2005) also find a radial anisotropy. Their spherically averaged $\beta$ of about 0.4 corresponds to a $\sigma_{\text {radial }} / \sigma_{\text {tangential }}$ of 1.3 , which is larger than our results along the major and minor axes. However, their simulations show declining projected dispersion profiles, which our data do not, that could account for the difference.

By modeling only the central part of NGC 821 , Gebhardt et al. (2003) find $M / L_{V}=7.6$ (without including foreground extinction), which is consistent with our no-halo value over the whole galaxy of $M / L_{V}=7.25$. Correcting for NGC 821's large reddening of $A_{V}=0.364 \mathrm{mag}$ (Schlegel et al. 1998, NED extragalactic database), we find our best-fitted $M / L_{V \text {, nohalo }}=$ 5.18, $M / L_{V, \mathrm{NFW}}=4.43$, and $M / L_{V, \text { pow }}=4.47$. Cappellari et al. (2006) find $M / L_{\text {jeans }}=3.54, M / L_{\text {schwarzschild }}=3.08$, and $M / L_{\text {stellar pop }}=2.60$ in the $I$ band. Using $(V-I)=1.35 \mathrm{mag}$ (Lauer et al. 2005), dereddened to $(V-I)_{0}=1.20 \mathrm{mag}$, and $(V-I)_{\odot}=0.682$ mag (Ramírez \& Meléndez 2005), our $V$-band $M / L$ s are converted to $M / L_{I, \text { no halo }}=3.21, M / L_{I, \mathrm{NFW}}=2.75$, and $M / L_{I, \text { pow }}=2.77$. Our $M / L$ s are slightly higher, though roughly consistent, with their $M / L$ s found using Schwarzschild modeling and stellar populations.

We find that the enclosed mass of NGC 821 within $\sim 2 R_{e}$ is roughly $2 \times 10^{11} M_{\odot}$, equally divided between stars and dark matter. At $1 R_{e}$, the ratio of dark matter halo mass to total mass is 0.19 for the best-fitted NFW halo profile and 0.13 for the best-fitted power-law halo profile. This matches other studies that find that the dark matter is $10 \%-40 \%$ of the total matter at $1 R_{e}$ and that dark matter begins to dominate at $2-4 R_{e}$ (e.g., Saglia et al. 2000; Gerhard et al. 2001; Mamon \& Łokas 
Table 5

Smoothing Model Results

\begin{tabular}{cccccc}
\hline \hline Halo & Smoothing & $\chi^{2}$ & $\begin{array}{c}r_{s} \\
(\mathrm{kpc})\end{array}$ & $c$ & $\begin{array}{c}\rho \\
\left(M_{\odot} \mathrm{pc}^{-3}\right) \\
(6)\end{array}$ \\
\hline None & $(2)$ & $(3)$ & $(4)$ & $(5)$ & $\ldots$ \\
None & No & 940.857 & $\ldots$ & $\ldots$ & $\ldots$ \\
NFW & Yes & 1033.73 & $\ldots$ & $\ldots$ & $\ldots$ \\
NFW & Yes & 766.47 & 1050 & 2.32 & $1.14 \times 10^{-4}$ \\
\hline
\end{tabular}

Notes. (1) Dark halo density profile. (2) Smoothing or no smoothing. (3) $\chi^{2}$ of best-fitted model. These $\chi^{2}$ values are lower than those of Table 4 because the models fit fewer data points (HET data only) than the models in Table 4 (HET and SAURON). (4) Scale radius of best-fitted model. (5) NFW concentration parameter determined from scale density. (6) NFW scale density $\rho_{\text {crit }} \delta_{c}$.

2005). The simulations of Dekel et al. (2005) also show that dark matter and stellar matter are equal at $3 R_{e}$ and at $1 R_{e}$ have a mass fraction of $40 \%$ dark matter. Thomas et al. (2007) perform similar dynamical modeling on 17 galaxies in the Coma cluster. Using values taken by eye from their Figure 5, we find that their average dark matter fraction at $1 R_{e}$ is 0.19 , though their galaxies show a wide range of dark matter fractions, from about 0.1 to 0.5 at $1 R_{e}$. We therefore find that the dark matter fraction at $1 R_{e}$ is similar for N821, a field elliptical galaxy, and a selection of Coma cluster early-type galaxies, perhaps contrary to hypotheses that environment plays a role in the dark matter fraction.

Weijmans et al. (2009) provide a dynamical analysis using orbit-based models and using data that extend to similar radii, though they do not attempt to characterize the shape of the halo. Thus, the comparison of dark halo mass results is informative. We find very similar numbers. Inside of $39^{\prime \prime}$ (which they call $R_{e}$ ), they find a dark matter fraction of $18 \%$. Inside of $45^{\prime \prime}$ (which we call $R_{e}$ ), we have a dark matter fraction of $13 \%$. There are differences in the models as well. First, they use a "maximum $M / L$ " model where they force the $M / L$ of the stars to have a maximum value. We find the best-fit stellar $M / L$ among the range modeled. Second, the modeling codes are different, with the main difference in that they use regularization (which trades the best-fitted values with smoothness) and we report results for the best fit to the data. Third, they use SAURON data at large radii and we use our HET data at large radii. Their data extend to slightly larger radii $\left(110^{\prime \prime}\right.$ compared to our limit of $\left.90^{\prime \prime}\right)$, and our data are high $\mathrm{S} / \mathrm{N}$. Given all of the these differences, it is impressive that we obtain similar results for the dark halo mass. This implies that systematic differences are not significant for determining the enclosed mass profile.

\subsection{Smoothing}

It is useful to constrain the orbital weighting so that the resulting DF is smooth, as a real galaxy's DF may be presumed to be. Although we do not usually report results when smoothing our models (we argue that allowing the best fit to the data is the most robust way to provide an un-biased result), other groups suggest that it is important for their model. Rix et al. (1997) and subsequent studies minimize the variation in the DF, a process they term regularization. We employ maximum entropy to find the best combination of orbit weights to match the data, as described in Thomas et al. (2005). We define a function $f \equiv \chi^{2}-\alpha S$, where $\chi^{2}$ is the sum of squared residuals to the data, $S$ is the entropy, and $\alpha$ is a parameter describing the relative weights of entropy and residuals in the fit. In order to minimize

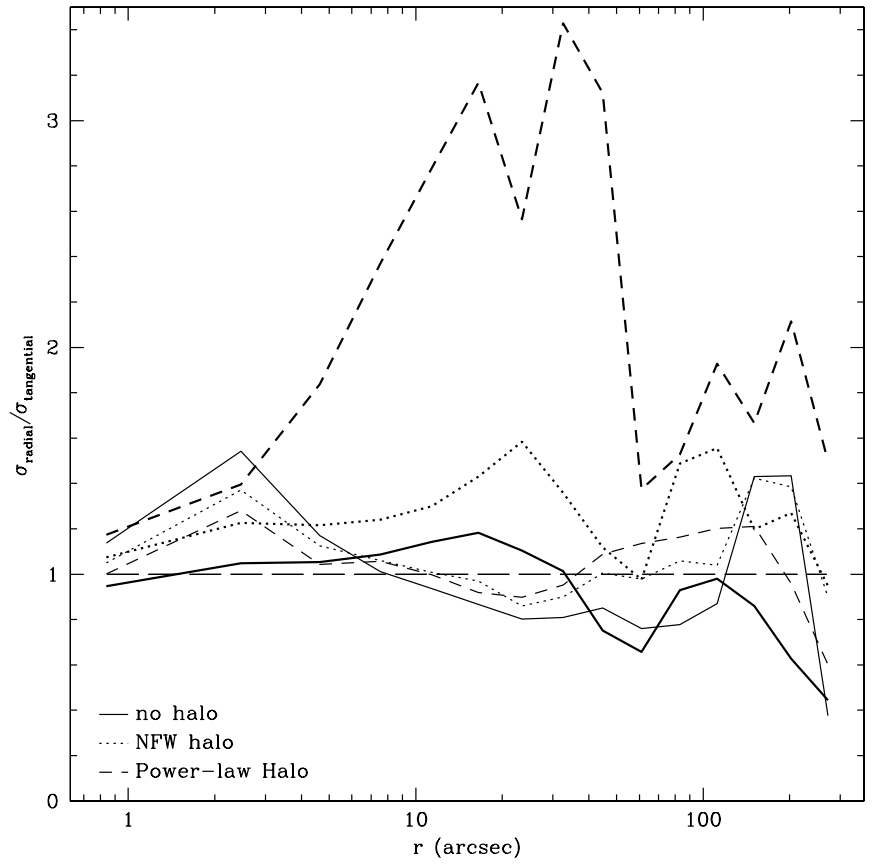

Figure 16. Ratio of radial to tangential dispersion (an average of $\sigma_{\theta}$ and $\sigma_{\phi}$, including streaming motion) along the major axis for models with only planetary nebula data (thick lines) and the best-fitted no halo, NFW halo, and power-law halo derived from the stellar kinematics. All three models are consistent with the PN data, in terms of $\chi^{2}$, with the dark halo models providing a slightly better fit. Thin lines show results for stellar data as comparison.

$f$, we typically start with a large value of $\alpha$ and make it smaller until the $\chi^{2}$ no longer varies. To test the effect of smoothing, we run models such that the iterations stop when $\alpha=0.01$, a reasonable value based on Thomas et al. (2005).

Using only our HET data, we ran our no-halo and NFWhalo models with and without smoothing using a coarser grid in parameter space. We find that smoothing does not alter the results. All of the models have a lower $\chi^{2}$ using only the HET data than the main results of our paper which use both HET and SAURON data. The models with smoothing have a larger $\chi^{2}$ than without smoothing (see Table 5), but the $\Delta \chi^{2}$ between different halo models remains the same. The best-fitted NFW dark halo parameters are consistent within the errors. The internal moments are also consistent with the unsmoothed models within the errors. Using an estimate by eye, the smoothed model's DF (plotted as $I_{3}$ versus $L_{z}$ in $E$ bins) looks similar to the unsmoothed model's DF when smoothed.

We therefore determine that adding smoothing via maximum entropy does not alter the measured halo, internal moments, or overall DF shape. We also note that these results using only our HET data are consistent with those using both HET and SAURON presented throughout this paper.

\subsection{Planetary Nebula Data}

We model the NGC 821 planetary nebula data of Romanowsky et al. (2003) with the best-fitted halos from the stellar data. We are not trying to constrain models using this data, but rather are interested in what orbital properties the planetary nebulae would require given the potential derived from the stellar data. In doing this, we assume that the potential derived from stars is correct and that the planetary nebulae are distributed in the same way as the stars. This assumption may not be realistic, as Dekel et al. (2005) predict that it is the densities, not the anisotropies, that differ. Figure 16 shows the ratio of radial to 
tangential dispersion for the models with no dark halo and bestfitted NFW and power-law halos. As expected from the results of Romanowsky et al. (2003), the model with no dark halo is roughly isotropic throughout and tangential at large radii. The best-fitted NFW model requires radial orbits throughout and the best-fitted power-law halo requires extremely radial orbits, with $\sigma_{\text {radial }} / \sigma_{\text {tangential }}$ of over 3 (corresponding to a $\beta$ of 0.9 ). This again demonstrates the strong mass-anisotropy degeneracy in dynamical studies. All three models are an excellent fit to the data, although there is a preference for a dark halo, but it is not statistically significant.

\section{CONCLUSIONS}

We present kinematics of NGC 821 to over 2 effective radii using long-slit spectroscopy from the HET and find that our measured stellar LOSVDs are larger than the planetary nebulae measurements of Romanowsky et al. (2003) at large radii.

Regardless of the density profile used, we are able to constrain the enclosed mass of NGC 821 within our kinematic data $\left(\sim 2 R_{e}\right)$ as roughly $2 \times 10^{11} M_{\odot}$, equally divided between stars and dark matter. At $1 R_{e}$ the ratio of dark matter halo mass to total mass is 0.19 for the best-fitted NFW halo profile and 0.13 for the best-fitted power-law halo profile.

We find that the best-fitted model of the dark halo in NGC 821 has a nearly flat power-law density profile. This dark halo gives a better fit than both the NFW halo models and models without a dark halo at a greater than $99 \%$ confidence level. This slope is somewhat unexpected and is strongly inconsistent with halo profiles with inner slopes greater than one (e.g., isothermal; Hernquist 1990; Moore et al. 1999) and may lend support to halos with a flat inner slope (e.g., cored isothermal, logarithmic potential, and Burkert 1995). Additionally, one would expect that adiabatic contraction would create even steeper inner halo profiles (Blumenthal et al. 1986; Gnedin et al. 2004), which is in conflict with our result. This halo result is driven by the data at large radii.

Our NFW $\chi^{2}$ space shows a degeneracy in radius and density as expected. This degeneracy is slightly tilted from the expected NFW correlations. Constraining these NFW radius and density parameters using a single concentration parameter could lead to biased results.

In addition to having a significantly poorer fit, the models without a dark halo show tangential anisotropy at large radii. This may be an indication that a dark halo is necessary because the radial component of the velocity dispersion may need to be artificially decreased at large radii in order to create a smaller total velocity dispersion that can be reproduced by a haloless model. The best-fitted dark halo model shows a radial bias in the $\theta$ direction at all radii. However, we do show that the velocities in the $\phi$ direction are greater than the radial component. If the planetary nebulae are on radial orbits, that would explain why our measured stellar velocity dispersions are larger than the reported planetary nebulae dispersions (Romanowsky et al. 2003). We show this by modeling the planetary nebula data assuming the potential of our best-fitted halo models from the stellar kinematics. We find that the planetary nebulae do require radially anisotropic orbits to match the best-fitted halo potentials.

The authors thank Tim de Zeeuw for his many helpful comments and discussions regarding the manuscript. We are very grateful to David Fisher for providing the surface brightness profile. K.G. acknowledges the support of the
Texas Advanced Research Program under grant 003658-02432001 and NSF-CAREER grant AST03-49095. The authors acknowledge use of the computational resources at the University of Texas at Austin's Texas Advanced Computing Center (http://www.tacc.utexas.edu) for the research reported in this paper. The Hobby-Eberly Telescope (HET) is a joint project of the University of Texas at Austin, the Pennsylvania State University, Stanford University, Ludwig-Maximilians-Universität München, and Georg-August-Universität Göttingen. The HET is named in honor of its principal benefactors, William P. Hobby and Robert E. Eberly.

\section{REFERENCES}

Barth, A. J., Ho, L. C., \& Sargent, W. L. W. 2002, AJ, 124, 2607

Beers, T. C., Flynn, K., \& Gebhardt, K. 1990, AJ, 100, 32

Bender, R., Doebereiner, S., \& Moellenhoff, C. 1988, A\&AS, 74, 385

Blumenthal, G. R., Faber, S. M., Flores, R., \& Primack, J. R. 1986, ApJ, 301, 27

Blumenthal, G. R., Faber, S. M., Primack, J. R., \& Rees, M. J. 1984, Nature, 311,517

Bullock, J. S., Kolatt, T. S., Sigad, Y., Somerville, R. S., Kravtsov, A. V., Klypin, A. A., Primack, J. R., \& Dekel, A. 2001, MNRAS, 321, 559

Burkert, A. 1995, ApJ, 447, L25

Cappellari, M., Verolme, E. K., van der Marel, R. P., Kleijn, G. A. V., Illingworth, G. D., Franx, M., Carollo, C. M., \& de Zeeuw, P. T. 2002, ApJ, 578, 787 Cappellari, M., et al. 2006, MNRAS, 366, 1126

Cappellari, M., et al. 2007, MNRAS, 379, 418

Coccato, L., et al. 2009, MNRAS, 394, 1249

Cretton, N., de Zeeuw, P. T., van der Marel, R. P., \& Rix, H.-W. 1999, ApJS, 124,383

Dekel, A., Stoehr, F., Mamon, G. A., Cox, T. J., Novak, G. S., \& Primack, J. R. 2005, Nature, 437, 707

de Vaucouleurs, G., de Vaucouleurs, A., Corwin, H. G., Jr., Buta, R. J., Paturel, G., \& Fouque, P. 1991, Third Reference Catalogue of Bright Galaxies Vols. 1-3, XII (Berlin: Springer)

Emsellem, E., et al. 2004, MNRAS, 352, 721

Emsellem, E., et al. 2007, MNRAS, 379, 401

Faber, S. M., Wegner, G., Burstein, D., Davies, R. L., Dressler, A., Lynden-Bell, D., \& Terlevich, R. J. 1989, ApJS, 69, 763

Freedman, W. L., et al. 2001, ApJ, 553, 47

Gebhardt, K., \& Thomas, J. 2009, ApJ, 700, 1690

Gebhardt, K., et al. 1996, AJ, 112, 105

Gebhardt, K., et al. 2000, AJ, 119, 1157

Gebhardt, K., et al. 2003, ApJ, 583, 92

Gerhard, O., Kronawitter, A., Saglia, R. P., \& Bender, R. 2001, AJ, 121, 1936

Gnedin, O. Y., Kravtsov, A. V., Klypin, A. A., \& Nagai, D. 2004, ApJ, 616, 16

Governato, F., Willman, B., Mayer, L., Brooks, A., Stinson, G., Valenzuela, O., Wadsley, J., \& Quinn, T. 2007, MNRAS, 374, 1479

Hernquist, L. 1990, ApJ, 356, 359

Hill, G. J., Nicklas, H. E., MacQueen, P. J., Tejada, C., Cobos Duenas, F. J., \& Mitsch, W. 1998, Proc. SPIE, 3355, 375

Houghton, R. C. W., Magorrian, J., Sarzi, M., Thatte, N., Davies, R. L., \& Krajnović, D. 2006, MNRAS, 367, 2

Keeton, C. R. 2001, ApJ, 561, 46

Kleyna, J., Wilkinson, M. I., Evans, N. W., Gilmore, G., \& Frayn, C. 2002, MNRAS, 330, 792

Komatsu, E., et al. 2009, ApJS, 180, 330

Lauer, T. R. 1985, MNRAS, 216, 429

Lauer, T. R., et al. 2005, AJ, 129, 2138

Leitherer, C., et al. 1996, PASP, 108, 996

Loewenstein, M., \& White, R. E., III 1999, ApJ, 518, 50

Macchetto, F., Pastoriza, M., Caon, N., Sparks, W. B., Giavalisco, M., Bender, R., \& Capaccioli, M. 1996, A\&AS, 120, 463

Magorrian, J. 2006, MNRAS, 373, 425

Mamon, G. A., \& Łokas, E. L. 2005, MNRAS, 363, 705

Mandelbaum, R., van de Ven, G., \& Keeton, C. R. 2009, MNRAS, 398, 635

Mateo, M. L. 1998, ARA\&A, 36, 435

Mathews, W. G., \& Brighenti, F. 2003, ARA\&A, 41, 191

Méndez, R. H., Riffeser, A., Kudritzki, R.-P., Matthias, M., Freeman, K. C., Arnaboldi, M., Capaccioli, M., \& Gerhard, O. E. 2001, ApJ, 563, 135

Moore, B., Quinn, T., Governato, F., Stadel, J., \& Lake, G. 1999, MNRAS, 310, 1147 
Naab, T., Johansson, P. H., Ostriker, J. P., \& Efstathiou, G. 2007, ApJ, 658, 710 Navarro, J. F., Frenk, C. S., \& White, S. D. M. 1996, ApJ, 462, 563

Ostriker, J. P., \& Steinhardt, P. 2003, Science, 300, 1909

Pellegrini, S., Baldi, A., Kim, D. W., Fabbiano, G., Soria, R., Siemiginowska, A., \& Elvis, M. 2007a, ApJ, 667, 731

Pellegrini, S., Siemiginowska, A., Fabbiano, G., Elvis, M., Greenhill, L., Soria, R., Baldi, A., \& Kim, D. W. 2007b, ApJ, 667, 749

Persic, M., Salucci, P., \& Stel, F. 1996, MNRAS, 281, 27

Pierce, M., et al. 2006, MNRAS, 366, 1253

Pinkney, J., et al. 2003, ApJ, 596, 903

Proctor, R. N., Forbes, D. A., Forestell, A., \& Gebhardt, K. 2005, MNRAS, 362 , 857

Proctor, R. N., Forbes, D. A., Romanowsky, A. J., Brodie, J. P., Strader, J., Spolaor, M., Mendel, J. T., \& Spitler, L. 2009, MNRAS, 398, 91

Ramírez, I., \& Meléndez, J. 2005, ApJ, 626, 465

Ravindranath, S., Ho, L. C., Peng, C. Y., Filippenko, A. V., \& Sargent, W. L. W. 2001, AJ, 122, 653

Richstone, D. O., \& Tremaine, S. 1988, ApJ, 327, 82

Rix, H.-W., de Zeeuw, P. T., Cretton, N., van der Marel, R. P., \& Carollo, C. M. 1997, ApJ, 488, 702

Romanowsky, A. J., Douglas, N. G., Arnaboldi, M., Kuijken, K., Merrifield, M. R., Napolitano, N. R., Capaccioli, M., \& Freeman, K. C. 2003, Science, 301, 1696
Saglia, R. P., Kronawitter, A., Gerhard, O., \& Bender, R. 2000, AJ, 119, 153 Sarzi, M., et al. 2006, MNRAS, 366, 1151

Schlegel, D. J., Finkbeiner, D. P., \& Davis, M. 1998, ApJ, 500, 525

Schwarzschild, M. 1979, ApJ, 232, 236

Sofue, Y., \& Rubin, V. 2001, ARA\&A, 39, 137

Thomas, J., Saglia, R. P., Bender, R., Thomas, D., Gebhardt, K., Magorrian, J., Corsini, E. M., \& Wegner, G. 2005, MNRAS, 360, 1355

Thomas, J., Saglia, R. P., Bender, R., Thomas, D., Gebhardt, K., Magorrian, J., Corsini, E. M., \& Wegner, G. 2007, MNRAS, 382, 657

Thomas, J., Saglia, R. P., Bender, R., Thomas, D., Gebhardt, K., Magorrian, J., \& Richstone, D. 2004, MNRAS, 353, 391

Tonry, J. L., Dressler, A., Blakeslee, J. P., Ajhar, E. A., Fletcher, A. B., Luppino, G. A., Metzger, M. R., \& Moore, C. B. 2001, ApJ, 546, 681

Trager, S. C., Faber, S. M., Worthey, G., \& González, J. J. 2000, AJ, 120, 165

van Albada, T. S., Bahcall, J. N., Begeman, K., \& Sancisi, R. 1985, ApJ, 295, 305

van der Marel, R. P., Cretton, N., de Zeeuw, P. T., \& Rix, H.-W. 1998, ApJ, 493, 613

Verolme, E. K., et al. 2002, MNRAS, 335, 517

Weijmans, A., et al. 2009, MNRAS, 398, 561

White, S. D. M., \& Rees, M. J. 1978, MNRAS, 183, 341

Zepf, S. E., Beasley, M. A., Bridges, T. J., Hanes, D. A., Sharples, R. M., Ashman, K. M., \& Geisler, D. 2000, AJ, 120, 2928 